\title{
Incrementally small-deformation theory for nonlinear analysis of structural frames
}

\author{
Y.B. Yang ${ }^{\text {a, }}$, S.R. Kuo ${ }^{\text {b }}$, Y.S. Wu ${ }^{\text {a }}$ \\ ${ }^{a}$ Department of Civil Engineering, National Taiwan University, Taipei, 10617 Taiwan, ROC \\ ${ }^{\mathrm{b}}$ Department of Harbor and River Engineering, National Taiwan Ocean University, Keelung, 20224 Taiwan, ROC
}

Received 7 November 2001; received in revised form 3 December 2001; accepted 19 December 2001

\begin{abstract}
An incrementally small-deformation theory that is physically self-explainable is presented for the large-displacement nonlinear analysis of structural frames. Strictly based on the assumption of small strains, small rotations, and small displacements within each incremental step, the elastic and geometric stiffness matrices for the beam element are derived from the force-displacement relations. Due consideration is taken of the 3D rotational behavior of nodal moments. The geometric stiffness matrix derived for the element is asymmetric. However, by enforcing all the joints to remain in equilibrium in the deformed configuration, the antisymmetric parts of the geometric stiffness matrices cancel out, resulting in a symmetric stiffness matrix for the structure. Also described is the procedure for updating the element forces and geometry in an incremental-iterative analysis. The present approach in its entire set is demonstrated to be robust and efficient for solving the nonlinear, postbuckling response of structural frames. (C) 2002 Elsevier Science Ltd. All rights reserved.
\end{abstract}

Keywords: Nonlinear analysis; Structural frame; Postbuckling analysis; Rigid body

\section{Introduction}

Historically, beam and truss elements are of pedagogical value to the instruction of the finite element methods, simply because their stiffness matrices can be directly derived from the force-displacement relations well known in structural mechanics. They serve as a bridge for linking the fundamental mechanics equations to the finite element matrix equations, at least for students starting to learn the matrix methods of structural analysis. However, as we proceed from linear to nonlinear domains, we find ourselves relying much more on advanced mechanics theories and numerical methods, not all of which are well covered by courses of advanced level. Obviously, nonlinear analysis is not a direct extension of linear analysis.

Unlike most previous works on nonlinear analysis, this paper is aimed at presenting a methodologically new

* Corresponding author. Tel.: +886-2-2363-2104; fax: +886-22363-7585.

E-mail address: ybyang@ccms.ntu.edu.tw (Y.B. Yang). and physically interpretable approach for formulating the beam element, as well as for conducting the nonlinear analysis. Clear physical meanings will be revealed in the formulation using the equilibrium approach, referred to as the direct approach, by adhering strictly to the assumption of small strains, small displacements, and small rotations for each incremental step of the nonlinear analysis. In particular, the elastic and geometric stiffness matrices for the 3D beam element will be derived directly from the force-displacement relations of the incremental theory. Such an approach is tutorially attractive, since all the terms involved are explicitly given and can be manipulated by hand. The geometric stiffness matrix derived is more compact than the conventional ones. Yet it contains the most essential properties that must be possessed by matrices of similar functions. For the 2D beam element, the elastic and geometric stiffness matrices are presented as a special case.

Issues that have been raised in the past concerning the nonlinear analysis of structural frames include the lack of equilibrium for structural joints connecting nonaligned members in the deformed configuration. This 
problem arises when the beam element is derived using established methods considering only a single, discrete member, based on the stress resultant definitions for bending moments and torques. Such an approach does not guarantee that the conditions of equilibrium be satisfied at the joints connecting non-aligned members in the deformed configuration. The reason is that the element stiffness matrices have been conventionally assembled with reference to the initial configuration of the structure, implying that equilibrium of the structure is satisfied only for the initial configuration, but not for the deformed configuration [1,2]. For the force quantities whose magnitude and direction remain unchanged upon rotations in space, such as the axial and shear forces, the conditions of equilibrium will be maintained in the deformed configuration. But for the others which may induce additional components upon 3D rotations, such as the bending moments and torques, the conditions of equilibrium will be violated in the deformed configuration.

Conventionally, a bending moment is interpreted as a quasitangential (QT) moment, and a torque as a semitangential (ST) moment, as they are generated as stress resultants over a cross section. By forcing the bending moments to behave in a semitangential way, Argyris et al. $[3,4]$ derived a $3 \mathrm{D}$ solid beam element that ensures equilibrium of the structure in the deformed configuration. To account for the effect of joint equilibrium in the rotated position, the concept of generalized rotations and moments, rather than the conventional rotations and moments, was adopted by Elias in formulating a nonlinear thin-walled beam element [5]. The definition of ST moment was adopted by Yang and McGuire in formulating a bisymmetric thin-walled beam element for geometric nonlinear analysis [6,7]. In the literature, numerous other works follow on this topic, which unfortunately have to be skipped in order to keep the paper within a reasonable length.

Another issue crucial to the geometric nonlinear analysis of structures is the way for performing iterations at each incremental step. Two key phases can be identified herein, i.e., the predictor or the solution of structural equations, and the corrector or the recovery of element forces. By taking advantage of the different roles of the two phases in each iteration, the present procedure in its entity is demonstrated to be both accurate and efficient for tracing the nonlinear, postbuckling response of structural frames. In order to highlight the procedural aspects of the theory, the effect of cross-sectional warping is just excluded.

\section{Formulation of the incremental theory}

Consider a cantilever subject to loadings of increasing magnitudes in Fig. 1. Three configurations can be ident-

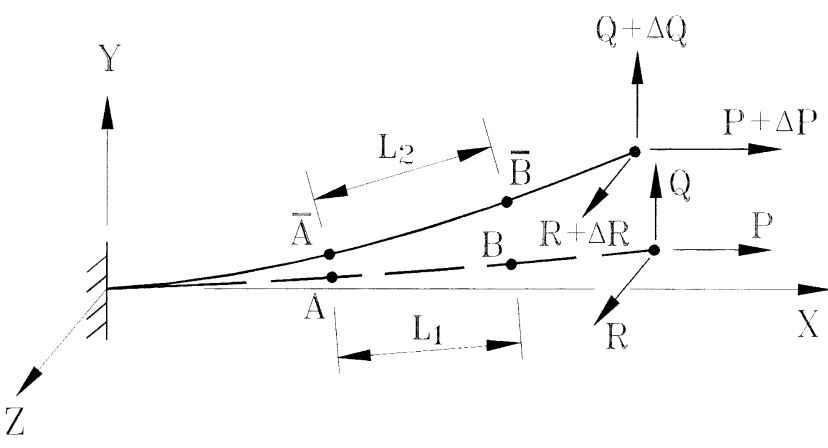

Fig. 1. Cantilever subjected to loads of increasingly magnitude.

ified for the cantilever, i.e., the initial (unloaded) $C_{0}$ configuration, the last (known) configuration $C_{1}$, and the current (unknown) configuration $C_{2}$. Using the updated Lagrangian (UL) formulation, the incremental equations of equilibrium will be derived for the beam element at $C_{2}$, but with reference to the $C_{1}$ configuration. The left superscript of a quantity denotes the configuration of occurrence. Force and displacement increments generated during the incremental step from $C_{1}$ to $C_{2}$ are denoted with no left superscript. Unless noted otherwise, a quantity with no left subscript is interpreted as one with reference to $C_{1}$ in the UL sense.

\subsection{Kinematics and displacements}

Let us focus on the incremental behavior of a typical element $A B$ of length $L_{1}(\equiv L)$ of the cantilever at $C_{1}$, which is initially under the action of the external loads $(P, Q, R)$, as shown in Fig. 1. Upon the increase of external loads from $(P, Q, R)$ to $(P+\Delta P, Q+\Delta Q, R+\Delta R)$, this element will be deformed into an element $\bar{A} \bar{B}$ of length $L_{2}$ at $C_{2}$. As shown in Fig. 2(a), we shall denote the longitudinal and two transverse axes of the element at $C_{1}$ by a set of orthogonal coordinates $x, y$ and $z$. Also, we shall denote the displacements of the two nodes $A$ and $B$ of the element from $C_{1}$ to $C_{2}$ by $\left(u_{A}, v_{A}, w_{A}\right)$ and $\left(u_{B}, v_{B}, w_{B}\right)$, respectively. The length $L_{2}$ of the element at the deformed configuration $C_{2}$ is

$L_{2}^{2}=\left(L+u_{B}-u_{A}\right)^{2}+\left(v_{B}-v_{A}\right)^{2}+\left(w_{B}-w_{A}\right)^{2}$

or

$L_{2} \cong L+u_{B}-u_{A}$

assuming the displacement increments to be small compared with the length of the element. The rigid displacements $u_{\mathrm{r}}, v_{\mathrm{r}}$ and $w_{\mathrm{r}}$ of the element at $C_{2}$ are

$$
\begin{gathered}
u_{\mathrm{r}}(x)=\frac{1}{2}\left(u_{A}+u_{B}\right) ; v_{\mathrm{r}}(x)=\frac{1}{2}\left(v_{A}+v_{B}\right) \\
+x \theta_{z \mathrm{r}} ; w_{\mathrm{r}}(x)=\frac{1}{2}\left(w_{A}+w_{B}\right)-x \theta_{y \mathrm{r}}
\end{gathered}
$$

Here, the angle of rigid twist $\theta_{x \mathrm{r}}$ of the element about 


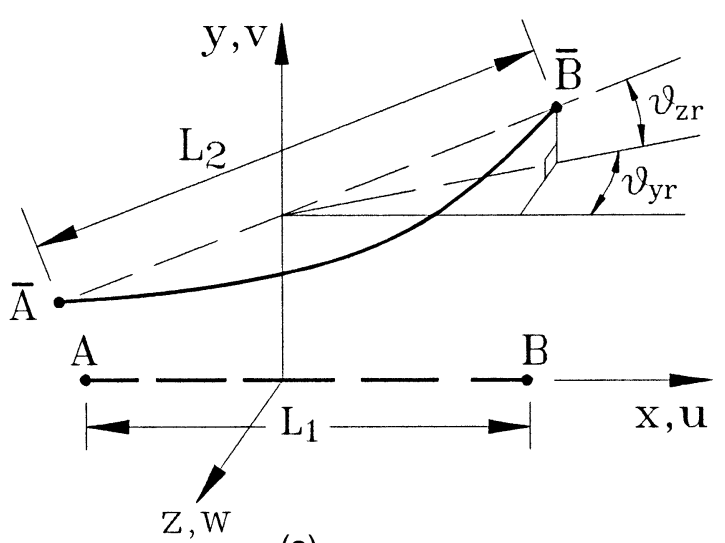

(a)

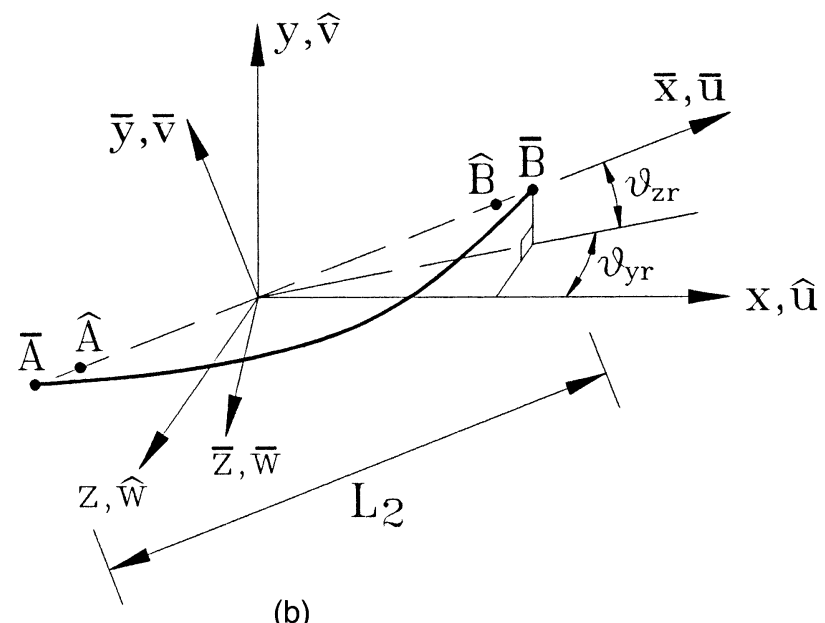

Fig. 2. Element $A B$ : (a) rigid displacement; (b) coordinates.

the $x$ axis and the rigid rotations $\theta_{y \mathrm{r}}$ and $\theta_{z \mathrm{r}}$ about the $y$ and $z$ axes are defined as

$\theta_{x \mathrm{r}}=\frac{1}{2}\left(\theta_{x A}+\theta_{x B}\right)$

$\theta_{y \mathrm{r}}=-\frac{1}{L}\left(w_{B}-w_{A}\right)$

$\theta_{z \mathrm{r}}=\frac{1}{L}\left(v_{B}-v_{A}\right)$

where $\theta_{x A}$ and $\theta_{x B}$ denote the angles of twist at the two ends of the element.

Let us now consider an intermediate state of the beam with only rigid translations and rigid rotations from $C_{1}$, but with no stretching, bending or twisting, namely, the $\hat{A} \hat{B}$ state in Fig. 2(b), where the axes $x, y$ and $z$ have translated from the $C_{1}$ state by an amount equal to the rigid translations. The axis $\bar{x}$ connecting the two nodes $\hat{A}$ and $\hat{B}$ of the element at $C_{2}$ can be related to the initial axis $x$ at $C_{1}$ as $\bar{x}=\left(L_{2} / L\right) x$. By deducting the rigid body components from the total displacements, the pure or natural deformations $(\bar{u}, \bar{v}, \bar{w})$ and angle of twist $\hat{\theta}_{x}$ of the element at $C_{2}$, as typified by the state $\bar{A} \bar{B}$, can be expressed with reference to the $C_{1}$ axes $(x, y, z)$ as

$$
\begin{aligned}
& \hat{u}(x)=u(x)-u_{\mathrm{r}}(x) ; \quad \hat{v}(x)=v(x)-v_{\mathrm{r}}(x) \\
& \hat{w}(x)=w(x)-w_{\mathrm{r}}(x) ; \quad \hat{\theta}_{x}(x)=\theta_{x}(x)-\theta_{x \mathrm{r}}
\end{aligned}
$$

Based on the assumption of small rotations for each incremental step, the preceding natural deformations and angle of twist can be transformed to the $(\bar{x}, \bar{y}, \bar{z})$ axes at $C_{2}$ as

$$
\begin{aligned}
& \left\{\begin{array}{l}
\bar{u}(x) \\
\bar{v}(x) \\
\bar{w}(x)
\end{array}\right\}=[\Delta R]\left\{\begin{array}{l}
\hat{u}(x) \\
\hat{v}(x) \\
\hat{w}(x)
\end{array}\right\} \\
& =\left[\begin{array}{lll}
1 & \theta_{z \mathrm{r}} & -\theta_{y \mathrm{r}} \\
-\theta_{z \mathrm{r}} & 1 & \theta_{x \mathrm{r}} \\
\theta_{y \mathrm{r}} & -\theta_{x \mathrm{r}} & 1
\end{array}\right]\left\{\begin{array}{l}
\hat{u}(x) \\
\hat{v}(x) \\
\hat{w}(x)
\end{array}\right\} \\
& \bar{\theta}_{x}(x)=\hat{\theta}_{x}(x)-\theta_{z \mathrm{r}} \frac{\partial \hat{w}}{\partial x}+\theta_{y \mathrm{r}} \frac{\partial \hat{v}}{\partial x}
\end{aligned}
$$

where $[\Delta R]$ is the rotation matrix and $-\partial \hat{w} / \partial x$ and $\partial \hat{v} / \partial x$ should be recognized as the natural rotations about the $y$ and $z$ axes, respectively.

\subsection{Hooke's law for the incremental step}

Consider the element during the incremental motion from $C_{1}$ to $C_{2}$. The nodal forces initially acting on the element at $C_{1}$ may be regarded as a set of forces in equilibrium, as shown in Fig. 3, assuming that iterations have been performed at each incremental step to remove the unbalanced forces. In response to the load increments from $C_{1}$ to $C_{2}$, the element will be further stressed, namely, subjected to both the initial nodal forces and the force increments generated during the step, as shown in Fig. 4. In the following, we shall focus on derivation of the relations between the force increments and displacement increments for the typical step from $C_{1}$ to $C_{2}$. By

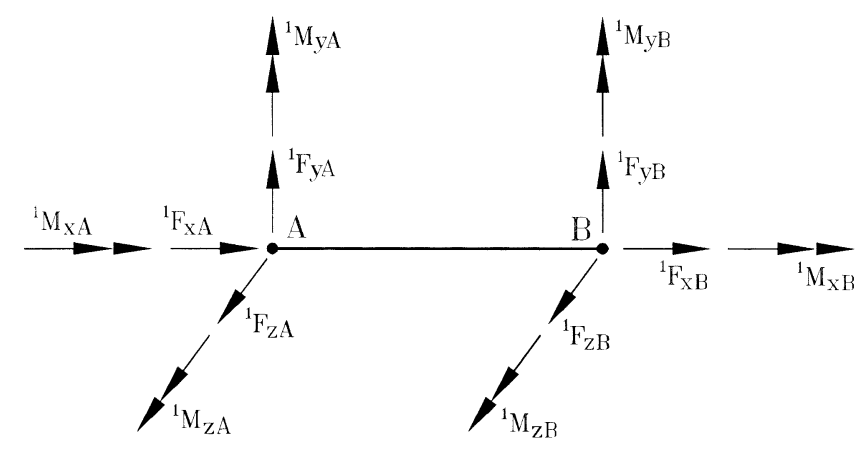

Fig. 3. Initial forces acting on the element at $C_{1}$. 


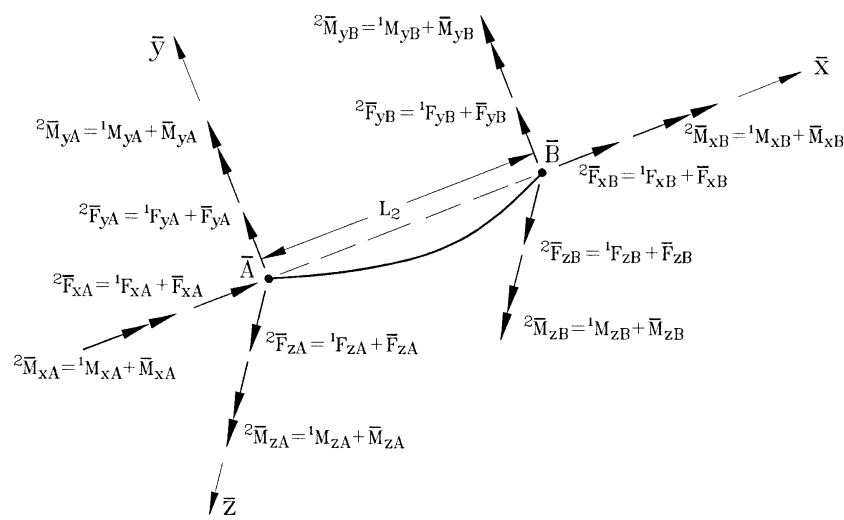

Fig. 4. Total forces acting on the element at $C_{2}$ but with reference to $C_{2}$.

the assumption of small strains for each incremental step, the axial force, torque, and moment increments caused by the natural deformation of the element from the state $\hat{A} \hat{B}$ to $\bar{A} \bar{B}$ are

$$
\begin{gathered}
N(x)=E A \frac{\mathrm{d} \bar{u}}{\mathrm{~d} \bar{x}}=E A \frac{L}{L_{2}} \frac{\mathrm{d} \bar{u}}{\mathrm{~d} x} \cong E A \frac{\mathrm{d} \hat{u}}{\mathrm{~d} x}=E A \frac{\mathrm{d} u}{\mathrm{~d} x} \\
M_{x}(x)=G J \frac{\mathrm{d} \bar{\theta}_{x}}{\mathrm{~d} \bar{x}}=G J \frac{L}{L_{2}} \frac{\mathrm{d} \bar{\theta}_{x}}{\mathrm{~d} x} \cong G J \frac{\mathrm{d} \hat{\theta}_{x}}{\mathrm{~d} x}=G J \frac{\mathrm{d} \theta_{x}}{\mathrm{~d} x} \\
M_{y}(x)=-E I_{y} \frac{\mathrm{d}^{2} \bar{w}}{\mathrm{~d} \bar{x}^{2}}=-E I_{y}\left(\frac{L}{L_{2}}\right)^{2} \frac{\mathrm{d}^{2} \bar{w}}{\mathrm{~d} x^{2}} \cong-E I_{y} \frac{\mathrm{d}^{2} \hat{w}}{\mathrm{~d} x^{2}} \\
=-E I_{y} \frac{\mathrm{d}^{2} w}{\mathrm{~d} x^{2}} \\
M_{z}(x)=E I_{z} \frac{\mathrm{d}^{2} \bar{v}}{\mathrm{~d} \bar{x}^{2}}=E I_{z}\left(\frac{L}{L_{2}}\right)^{2} \frac{\mathrm{d}^{2} \bar{v}}{\mathrm{~d} x^{2}} \cong E I_{z} \frac{\mathrm{d}^{2} \bar{v}}{\mathrm{~d} x^{2}}=E I_{z} \frac{\mathrm{d}^{2} v}{\mathrm{~d} x^{2}}
\end{gathered}
$$

where $E$ is Young's modulus, $G$ the shear modulus, $A$ the cross-sectional area, $J$ the torsional constant, and $I_{y}$ and $I_{z}$ moments of inertia of the element about the $y$ and $z$ axes, respectively.

\subsection{Nature of moments undergoing rotations}

For a conservative direct force, the direction and magnitude remain unchanged when the structure on which the force acts is displaced. Hence, a vector expression for a conservative direct force in the deformed configuration is identical to that in the initial configuration. However, the same is not true with conservative moments [3-9]. First, their direction and magnitude do not remain the same when the system on which they act undergoes rotations. Second, there are various kinds of conservative moments, which differ in the way they behave when undergoing rotations. Consequently, a vector expression for a conservative moment in the deformed configuration is generally different from that in the initial configuration.

Conventionally, the torque ${ }^{1} M_{x}$ at $C_{1}$ has been defined as the resultant of the initial shear stresses $\tau_{x z}$ and $\tau_{y z}$ integrated over the cross-sectional area $A$ of the beam, and the bending moments ${ }^{1} M_{y}$ and ${ }^{1} M_{z}$ as the resultants of the bending effects of the initial axial stress $\sigma_{x}$, that is,

$$
\begin{aligned}
{ }^{1} M_{x} & =\int_{A}\left(\tau_{z x} y-\tau_{y x} z\right) \mathrm{d} A ; \quad{ }^{1} M_{y}=\int_{A} \sigma_{x} z \mathrm{~d} A ; \quad{ }^{1} M_{z} \\
& =-\int_{A} \sigma_{x} y \mathrm{~d} A ;
\end{aligned}
$$

Based on the preceding definitions, Argyris, et al. [3,4] have shown that the torque ${ }^{1} M_{x}$ acting at $C_{1}$ is a semitangential (ST) moment, and the bending moments ${ }^{1} M_{y}$ and ${ }^{1} M_{z}$ are quasitangential (QT) moments, in the sense that the moments given in Eq. (9) will be induced when subjected to the $3 \mathrm{D}$ rotations $\theta_{x}, \theta_{y}$, and $\theta_{z}$ :

$$
\begin{aligned}
& \Delta M_{y}=\frac{1}{2} M_{x} \theta_{z} ; \quad \Delta M_{z}=-\frac{1}{2} M_{x} \theta_{y} \\
& \Delta M_{z}={ }^{1} M_{y} \theta_{x} ; \quad \Delta M_{y}=-{ }^{1} M_{z} \theta_{x}
\end{aligned}
$$

Note that the induced moments given in Eq. (9) are associated with the rigid rotations of the cross section on which the torque or moments act, which have nothing to do with straining or deformation of the element.

\subsection{Force increments generated during the incremental step}

The axial force, torque, and moment increments acting on the element at the state should be computed as the summation of the natural deformation and rigid rotation effects given in Eq. (7) and (9). Namely, by interpreting the rotations $\theta_{x}, \theta_{y}$ and $\theta_{z}$ in Eq. (9) as the rotation increments from $\hat{A} \hat{B}$ to $\bar{A} \bar{B}$, which can be obtained by deducting the rigid rotations from the nodal rotations, the axial force, torque, and moment increments at end $B$ (i.e., at $x=L$ ) of the element can be computed as the summation of the two effects given in Eq. (7) and (9), that is,

$$
\begin{aligned}
& \bar{F}_{x B}=E A u^{\prime}(L) \\
& \bar{M}_{x B}=G J \theta_{x}{ }^{\prime}(L) \\
& \bar{M}_{y B}=-E I_{y} w^{\prime \prime}(L)+\frac{1}{2} M_{x B}\left(\theta_{z B}-\theta_{z \mathrm{r}}\right)-{ }^{1} M_{z B}\left(\theta_{x B}\right. \\
& \left.\quad-\theta_{x \mathrm{r}}\right) \\
& \bar{M}_{z B}=E I_{z} v^{\prime \prime}(L)-\frac{1}{2} M_{x B}\left(\theta_{y B}-\theta_{y \mathrm{r}}\right)+{ }^{1} M_{y B}\left(\theta_{x B}\right. \\
& \left.-\theta_{x \mathrm{r}}\right)
\end{aligned}
$$

where $(\cdot)^{\prime}=\mathrm{d}(\cdot) / \mathrm{d} x$ and the axes of reference are those associated with the state $\bar{A} \bar{B}$ of the element at $C_{2}$. In the same manner, it is easy to show that the axial force, torque, and moment increments at end $A$, i.e., at $x=0$, of the element are 


$$
\begin{aligned}
& \bar{F}_{x A}=-E A u^{\prime}(0) \\
& \bar{M}_{x A}=-G J \theta_{x}^{\prime}(0) \\
& \bar{M}_{y A}=E I_{y} w^{\prime \prime}(0)+\frac{1}{2} M_{x A}\left(\theta_{z A}-\theta_{z \mathrm{r}}\right)-{ }^{1} M_{z A}\left(\theta_{x A}\right. \\
& \left.\quad-\theta_{x \mathrm{r}}\right) \\
& \bar{M}_{z A}=-E I_{z} v^{\prime \prime}(0)-\frac{1}{2}{ }^{1} M_{x A}\left(\theta_{y A}-\theta_{y \mathrm{r}}\right)+{ }^{1} M_{y A}\left(\theta_{x A}\right. \\
& \left.-\theta_{x \mathrm{r}}\right)
\end{aligned}
$$

Unlike the above force increments, the shear force increments $\bar{F}_{y B}$ and $\bar{F}_{z B}$ generated at $C_{2}$ cannot be obtained from Hooke's law. Rather, they should be obtained from the conditions of equilibrium, as implied by the Bernoulli-Euler hypothesis for beams. From Fig. 4 , the following equation of equilibrium can be written,

$$
\begin{aligned}
& \left({ }^{1} F_{y B}+\bar{F}_{y B}\right) L_{2}=-\left({ }^{1} M_{z B}+\bar{M}_{z B}\right)-\left({ }^{1} M_{z A}\right. \\
& \left.\quad+\bar{M}_{z A}\right)
\end{aligned}
$$

By the condition of equilibrium for the element at $C_{1}$ (Fig. 3),

$$
{ }^{1} F_{y B}=-\frac{1}{L}\left({ }^{1} M_{z B}+{ }^{1} M_{z A}\right)
$$

along with Eq. (2) for the element length $L_{2}$, the shear force increment $\bar{F}_{y B}$ can be derived from Eq. (12) as

$$
\begin{aligned}
\bar{F}_{y B} & \cong-\frac{1}{L}\left(\bar{M}_{z B}+\bar{M}_{z A}\right)+\frac{1}{L}\left({ }^{1} M_{z B}+{ }^{1} M_{z A}\right) \frac{u_{B}-u_{A}}{L} \\
& =-\frac{1}{L}\left(\bar{M}_{z B}+\bar{M}_{z A}\right)-{ }^{1} F_{y B} \frac{u_{B}-u_{A}}{L}
\end{aligned}
$$

Similarly, the shear force increment $\bar{F}_{z B}$ generated at $C_{2}$ can be computed as

$$
\begin{aligned}
\bar{F}_{z B} & \cong-\frac{1}{L}\left(\bar{M}_{y B}+\bar{M}_{y A}\right)-\frac{1}{L}\left({ }^{1} M_{y B}+{ }^{1} M_{y A}\right) \frac{u_{B}-u_{A}}{L} \\
& =\frac{1}{L}\left(\bar{M}_{y B}+\bar{M}_{y A}\right)-{ }^{1} F_{z B} \frac{u_{B}-u_{A}}{L}
\end{aligned}
$$

if use is made of the following relation:

$$
{ }^{1} F_{z B}=\frac{1}{L}\left({ }^{1} M_{y B}+{ }^{1} M_{y A}\right)
$$

Obviously, the shear force increments given in Eqs. (14) and (15) have been expressed with reference to the $C_{2}$ axes as well.

In the following, we shall transform the reference axes for all the force increments to those at $C_{1}$, to conform to the UL formulation. In Fig. 5, the total nodal forces acting on the element at $C_{2}$ have been expressed with reference to $C_{1}$. A comparison of Fig. 4 with Fig. 5 yields the relations for transforming the total nodal forces from the $C_{2}$ to the $C_{1}$ axes:

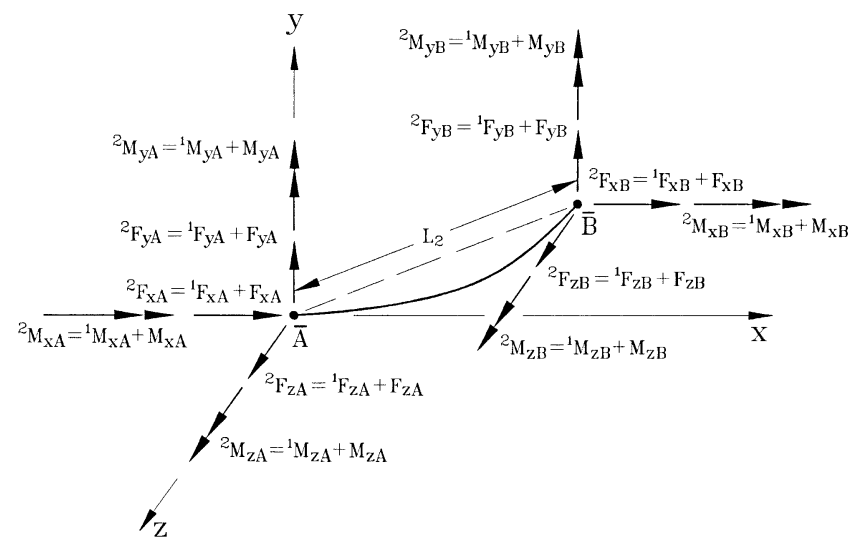

Fig. 5. Total forces acting on the element at $C_{2}$ and with reference to $C_{1}$.

$\left\{\begin{array}{c}{ }^{2} F_{x B} \\ { }^{2} F_{y B} \\ { }^{2} F_{z B}\end{array}\right\}=\left[\begin{array}{lll}1 & -\theta_{x \mathrm{r}} & \theta_{y \mathrm{r}} \\ \theta_{z \mathrm{r}} & 1 & -\theta_{x \mathrm{r}} \\ -\theta_{y \mathrm{r}} & \theta_{x \mathrm{r}} & 1\end{array}\right]\left\{\begin{array}{c}{ }^{2} \bar{F}_{x B} \\ { }^{2} \bar{F}_{y B} \\ { }^{2} \bar{F}_{z B}\end{array}\right\}$

By decomposing the total nodal forces as

$$
\begin{gathered}
\left\{\begin{array}{c}
{ }^{2} F_{x B} \\
{ }^{2} F_{y B} \\
{ }^{2} F_{z B}
\end{array}\right\}=\left\{\begin{array}{c}
{ }^{1} F_{x B}+F_{x B} \\
{ }^{1} F_{y B}+F_{y B} \\
{ }^{1} F_{z B}+F_{z B}
\end{array}\right\} ; \quad\left\{\begin{array}{c}
{ }^{2} \bar{F}_{x B} \\
{ }^{2} \bar{F}_{y B} \\
{ }^{2} \bar{F}_{z B}
\end{array}\right\} \\
=\left\{\begin{array}{c}
{ }^{1} F_{x B}+\bar{F}_{x B} \\
{ }^{1} F_{y B}+\bar{F}_{y B} \\
{ }^{1} F_{z B}+\bar{F}_{z B}
\end{array}\right\}
\end{gathered}
$$

and neglecting higher order terms, i.e., products of force and displacement increments, the axial and shear force increments with respect to the $C_{1}$ axes can be obtained from Eq. (17) as

$$
\left\{\begin{array}{l}
F_{x B} \\
F_{y B} \\
F_{z B}
\end{array}\right\}=\left\{\begin{array}{l}
\bar{F}_{x B} \\
\bar{F}_{y B} \\
\bar{F}_{z B}
\end{array}\right\}+\left[\begin{array}{lll}
0 & -\theta_{z \mathrm{r}} & \theta_{y \mathrm{r}} \\
\theta_{z \mathrm{r}} & 0 & -\theta_{x \mathrm{r}} \\
-\theta_{y \mathrm{r}} & \theta_{x \mathrm{r}} & 0
\end{array}\right]\left\{\begin{array}{c}
{ }^{1} F_{x B} \\
{ }^{1} F_{y B} \\
{ }^{1} F_{z B}
\end{array}\right\}
$$

Substituting Eqs. (4b) and (4c) for $\theta_{y \mathrm{r}}, \theta_{z \mathrm{r}}$, Eq. (10a) for $\bar{F}_{x B}$, Eq. (13) for ${ }^{1} F_{y B}$, and Eq. (16) for ${ }^{1} F_{z B}$, one can obtain from Eq. (19) the axial force increment $F_{x B}$ acting at node $B$ of the element as

$$
\begin{gathered}
F_{x B}=E A u^{\prime}(L)+\frac{{ }^{1} M_{z A}+{ }^{1} M_{z B}}{L^{2}}[v(L)-v(0)] \\
-\frac{{ }^{1} M_{y A}+{ }^{1} M_{y B}}{L^{2}}[w(L)-w(0)]
\end{gathered}
$$

where it is realized that $u_{A}=u(0), u_{B}=u(L)$, etc. The 
axial force increment $F_{x A}$ acting at node $A$ of the element is equal to $-F_{x B}$, that is,

$$
\begin{aligned}
F_{x A} & =-E A u^{\prime}(L)-\frac{{ }^{1} M_{z A}+{ }^{1} M_{z B}}{L^{2}}[v(L)-v(0)] \\
+ & \frac{{ }^{1} M_{y A}+{ }^{1} M_{y B}}{L^{2}}[w(L)-w(0)]
\end{aligned}
$$

At this point, we have related the axial force increments $F_{x A}$ and $F_{x B}$ to the displacement increments $u, v$ and $w$ of the element for the incremental step.

Substituting Eq. (4c) for $\theta_{z \mathrm{r}}$ and Eq. (14) for $\bar{F}_{y B}$, one can derive from Eq. (19) the shear force increment $F_{y B}$ acting at end $B$ of the element as

$$
\begin{gathered}
F_{y B}=-\frac{1}{L}\left(\bar{M}_{z B}+\bar{M}_{z A}\right)-\frac{{ }^{1} F_{y B}}{L}\left(u_{B}-u_{A}\right)+\frac{{ }^{1} F_{x B}}{L}\left(v_{B}\right. \\
\left.-v_{A}\right)-{ }^{1} F_{z B} \theta_{x \mathrm{r}}
\end{gathered}
$$

By substitution of Eq. (10d) for $\bar{M}_{z B}$, Eq. (11d) for $\bar{M}_{z A}$, Eq. (13) for ${ }^{1} F_{y B}$, and Eq. (16) for ${ }^{1} F_{z B}$, and noting that ${ }^{1} M_{x A}=-{ }^{1} M_{x B}$, the preceding equation can be manipulated to yield

$$
\begin{aligned}
& F_{y B}=-\frac{E I_{z}}{L}\left[v^{\prime \prime}(L)-v^{\prime \prime}(0)\right]+\frac{{ }^{1} M_{z A}+{ }^{1} M_{z B}}{L^{2}}[u(L) \\
& -u(0)]+\frac{{ }^{1} F_{x B}}{L}[v(L)-v(0)]+\frac{{ }^{1} M_{x B}}{2 L}\left[\theta_{y}(L)-\theta_{y}(0)\right] \\
& -\frac{{ }^{1} M_{y B}}{L} \theta_{x}(L)-\frac{{ }^{1} M_{y A}}{L} \theta_{x}(0)
\end{aligned}
$$

The shear force increment $F_{y A}$ acting at end $A$ of the element is equal to $-F_{y B}$, that is,

$$
\begin{aligned}
& F_{y A}=\frac{E I_{z}}{L}\left[v^{\prime \prime}(L)-v^{\prime \prime}(0)\right]-\frac{{ }^{1} M_{z A}+{ }^{1} M_{z B}}{L^{2}}[u(L) \\
& -u(0)]-\frac{{ }^{1} F_{x B}}{L}[v(L)-v(0)]-\frac{{ }^{1} M_{x B}}{2 L}\left[\theta_{y}(L)-\theta_{y}(0)\right] \\
& +\frac{{ }^{1} M_{y B}}{L} \theta_{x}(L)+\frac{{ }^{1} M_{y A}}{L} \theta_{x}(0)
\end{aligned}
$$

Similarly, the shear force increments $F_{z B}$ and $F_{z A}$ acting at the two ends of the element can be expressed as follows:

$$
\begin{aligned}
& F_{z B}=-\frac{E I_{y}}{L}\left[w^{\prime \prime}(L)-w^{\prime \prime}(0)\right]-\frac{{ }^{1} M_{y A}+{ }^{1} M_{y B}}{L^{2}}[u(L) \\
& -u(0)]+\frac{{ }^{1} F_{x B}}{L}[w(L)-w(0)]+\frac{{ }^{1} M_{x B}}{2 L}\left[\theta_{z}(L)\right. \\
& \left.-\theta_{z}(0)\right]-\frac{{ }^{1} M_{z B}}{L} \theta_{x}(L)-\frac{{ }^{1} M_{z A}}{L} \theta_{x}(0) \\
& F_{z A}=\frac{E I_{y}}{L}\left[w^{\prime \prime}(L)-w^{\prime \prime}(0)\right]+\frac{{ }^{1} M_{y A}+{ }^{1} M_{y B}}{L^{2}}[u(L) \\
& -u(0)]-\frac{{ }^{1} F_{x B}}{L}[w(L)-w(0)]-\frac{{ }^{1} M_{x B}}{2 L}\left[\theta_{z}(L)-\theta_{z}(0)\right] \\
& +\frac{{ }^{1} M_{z B}}{L} \theta_{x}(L)+\frac{{ }^{1} M_{z A}}{L} \theta_{x}(0)
\end{aligned}
$$

At this point, we have expressed all the force increments in terms of the initial nodal forces and displacement increments for the element stepping from $C_{1}$ to $C_{2}$. In the following, we shall focus on derivation of similar expressions for the moment increments. By comparing Fig. 4 with Fig. 5, it is easy to see that the relations given in Eqs. (17)-(19) for the force quantities remain equally valid for the moment quantities. Consequently, the moment increments at end $B$ of the element with respect to the $C_{1}$ axes, i.e., $M_{x B}, \ldots$, can be related to those of the $C_{2}$ axes, i.e., $M_{x B}, \ldots$, and the initial nodal moments ${ }^{1} M_{x B}, \ldots$, in a way similar to Eq. (19) as

$$
\left\{\begin{array}{l}
M_{x B} \\
M_{y B} \\
M_{z B}
\end{array}\right\}=\left\{\begin{array}{l}
\bar{M}_{x B} \\
\bar{M}_{y B} \\
\bar{M}_{z B}
\end{array}\right\}+\left[\begin{array}{lll}
0 & -\theta_{z r} & \theta_{y \mathrm{r}} \\
\theta_{z \mathrm{r}} & 0 & -\theta_{x \mathrm{r}} \\
-\theta_{y \mathrm{r}} & \theta_{x \mathrm{r}} & 0
\end{array}\right]\left\{\begin{array}{c}
{ }^{1} M_{x B} \\
{ }^{1} M_{y B} \\
{ }^{1} M_{z B}
\end{array}\right\}
$$

By substitution of Eqs. (4b) and (4c) for $\theta_{y \mathrm{r}}, \theta_{z \mathrm{r}}$, Eq. (10b) for $\bar{M}_{x B}$, one can derive from Eq. (27) the torque increment $M_{x B}$ for end $B$ of the element as

$$
\begin{aligned}
& M_{x B}=G J \theta_{x}{ }^{\prime}(L)-\frac{{ }^{1} M_{y B}}{L}[v(L)-v(0)]-\frac{{ }^{1} M_{z B}}{L}[w(L) \\
& \quad-w(0)]
\end{aligned}
$$

Next, by replacing the generalized strain $\theta^{\prime}{ }_{x}(L)$ by $-\theta^{\prime}{ }_{x}(0)$, the initial moment ${ }^{1} M_{y B}$ by ${ }^{1} M_{y A}$, and ${ }^{1} M_{z B}$ by ${ }^{1} M_{z A}$ in Eq. (28), the torque increment $M_{x A}$ for end $A$ can be written as

$$
\begin{aligned}
& M_{x A}=-G J \theta_{x}{ }^{\prime}(0)-\frac{{ }^{1} M_{y A}}{L}[v(L)-v(0)]-\frac{{ }^{1} M_{z A}}{L}[w(L) \\
& \quad-w(0)]
\end{aligned}
$$

By substitution of Eq. (4c) for $\theta_{z r}$, Eq. (10c) for $\bar{M}_{y B}$, the moment increment $M_{y B}$ for end $B$ of the element can be derived from Eq. (27) as

$$
\begin{gathered}
M_{y B}=-E I_{y} w^{\prime \prime}(L)+\frac{{ }^{1} M_{x B}}{2} \theta_{z}(L)+\frac{{ }^{1} M_{x B}}{2 L}[v(L) \\
-v(0)]-{ }^{1} M_{z B} \theta_{x}(L)
\end{gathered}
$$

By replacing the generalized strain $w^{\prime \prime}(L)$ by $-w^{\prime \prime}(0)$, the rotation $\theta_{z}(L)$ by $\theta_{z}(0), \theta_{x}(L)$ by $\theta_{x}(0)$, the initial moment ${ }^{1} M_{x B}$ by ${ }^{1} M_{x A}$ (which equals $-{ }^{1} M_{x B}$ ), and ${ }^{1} M_{z B}$ by ${ }^{1} M_{z A}$, the moment increment $M_{y A}$ associated with end $A$ of the element can be obtained from Eq. (30) as

$$
\begin{gathered}
M_{y A}=E I_{y} w^{\prime \prime}(0)-\frac{{ }^{1} M_{x B}}{2} \theta_{z}(0)-\frac{{ }^{1} M_{x B}}{2 L}[v(L)-v(0)] \\
-{ }^{1} M_{z A} \theta_{x}(0)
\end{gathered}
$$

In a similar way, the moment increments $M_{z B}$ for end $B$ of the element can be derived as

$$
\begin{aligned}
& M_{z B}=E I_{z} v^{\prime \prime}(L)-\frac{{ }^{1} M_{x B}}{2} \theta_{y}(L)+\frac{{ }^{1} M_{x B}}{2 L}[w(L)-w(0)] \\
& \quad+{ }^{1} M_{y B} \theta_{x}(L)
\end{aligned}
$$


and the moment increment $M_{z A}$ for end $A$ is

$$
\begin{gathered}
M_{z A}=-E I_{z} v^{\prime \prime}(0)+\frac{{ }^{1} M_{x B}}{2} \theta_{y}(0)-\frac{{ }^{1} M_{x B}}{2 L}[w(L) \\
-w(0)]+{ }^{1} M_{y A} \theta_{x}(0)
\end{gathered}
$$

Thus, we have expressed all the force and moment increments for the two ends of the element in terms of the initial nodal forces and displacement increments occurring for the incremental step from $C_{1}$ to $C_{2}$. The incremental force-displacement relations as given in Eqs. (20, 21, 23-26, 28-33) represent exactly the natural boundary conditions of the beam for the incremental theory.

\subsection{Element interpolation functions}

In the following, a square matrix, a column vector and a row vector will be enclosed by the symbols [], \{ $\}$ and \langle\rangle , respectively. Consider that the element is subjected only to external loads at the nodal points. Based on the assumption of small displacements within the incremental step, the differential equations of equilibrium for a cross section of the element at $C_{2}$, but with respect to the $C_{1}$ axes, can be written as

$$
\begin{aligned}
& E A \frac{\mathrm{d}^{2} \bar{u}}{\mathrm{~d} \bar{x}^{2}}=E A\left(\frac{L}{L_{2}}\right)^{2} \frac{\mathrm{d}^{2} \bar{u}}{\mathrm{~d} x^{2}} \cong E A \frac{\mathrm{d}^{2} \bar{u}}{\mathrm{~d} x^{2}} \cong E A \frac{\mathrm{d}^{2} \hat{u}}{\mathrm{~d} x^{2}}=E A \frac{\mathrm{d}^{2} u}{\mathrm{~d} x^{2}} \\
& =0 \\
& E I_{z} \frac{\mathrm{d}^{4} \bar{v}}{\mathrm{~d} \bar{x}^{4}}=E I_{z}\left(\frac{L}{L_{2}}\right)^{4} \frac{\mathrm{d}^{4} \bar{v}}{\mathrm{~d} x^{4}} \cong E I_{z} \frac{\mathrm{d}^{4} \bar{v}}{\mathrm{~d} x^{4}} \cong E I_{z} \frac{\mathrm{d}^{4} \hat{v}}{\mathrm{~d} x^{4}}=E I_{z} \frac{\mathrm{d}^{4} v}{\mathrm{~d} x^{4}}=0 \\
& E I_{y} \frac{\mathrm{d}^{4} \bar{w}}{\mathrm{~d} \bar{x}^{4}}=E I_{y}\left(\frac{L}{L_{2}}\right)^{4} \frac{\mathrm{d}^{4} \bar{w}}{\mathrm{~d} x^{4}} \cong E I_{y} \frac{\mathrm{d}^{4} \bar{w}}{\mathrm{~d} x^{4}} \cong E I_{y} \frac{\mathrm{d}^{4} \bar{w}}{\mathrm{~d} x^{4}}=E I_{y} \frac{\mathrm{d}^{4} w}{\mathrm{~d} x^{4}}=0 \\
& G J \frac{\mathrm{d}^{2} \bar{\theta}_{x}}{\mathrm{~d} \bar{x}^{2}}=G J\left(\frac{L}{L_{2}}\right)^{2} \frac{\mathrm{d}^{2} \bar{\theta}_{x}}{\mathrm{~d} x^{2}} \cong G J \frac{\mathrm{d}^{2} \bar{\theta}_{x}}{\mathrm{~d} x^{2}} \cong G J \frac{\mathrm{d}^{2} \bar{\theta}_{x}}{\mathrm{~d} x^{2}}=G J \frac{\mathrm{d}^{2} \theta_{x}}{\mathrm{~d} x^{2}} \\
& =0
\end{aligned}
$$

Obviously, by the assumptions of small strains, small rotations and small displacements for each incremental step, both the axial and torsional displacement increments, $u$ and $\theta_{x}$, can be represented by linear functions, and the transverse displacement increments $v$ and $w$ by cubic polynomial functions. Consequently,

$u=\langle(1-\xi) \xi\rangle\left\{u_{x}\right\}$

$v=\left\langle 1-3 \xi^{2}+2 \xi^{3} 3 \xi^{2}-2 \xi^{3} \xi L(1-\xi)^{2} \xi^{2} L(\xi-1)\right\rangle\left\{u_{y}\right\}$

$w=\left\langle 1-3 \xi^{2}+2 \xi^{3} 3 \xi^{2}-2 \xi^{3} \xi L(1-\xi)^{2} \xi^{2} L(\xi-1)\right\rangle\left\{u_{z}\right\}$

$\theta_{x}=\langle(1-\xi) \xi\rangle\left\{\theta_{x}\right\}$

where $\xi=x / L,\left\{u_{x}\right\}$ and $\left\{\theta_{x}\right\}$ respectively denote the nodal vector containing the axial and torsional displacement degrees of freedom (DOFs), and $\left\{u_{y}\right\}$ and $\left\{u_{z}\right\}$ respectively the nodal vector containing the DOFs associated with $y$ - and $z$-direction displacements,

$$
\begin{aligned}
& \left\{u_{x}\right\}^{T}=\left\langle u_{A} u_{B}\right\rangle\left\{\theta_{x}\right\}^{T}=\left\langle\theta_{x A} \theta_{x B}\right\rangle \\
& \left\{u_{y}\right\}^{T}=\left\langle v_{A} v_{B} \theta_{z A} \theta_{z B}\right\rangle\left\{u_{z}\right\}^{T}=\left\langle w_{A} w_{B}-\theta_{y A}-\theta_{y B}\right\rangle
\end{aligned}
$$

By substituting the interpolation functions for $u, v, w$ and $\theta_{x}$, and noting that $\theta_{y}=-w^{\prime}$ and $\theta_{z}=v^{\prime}$, one can derive from the incremental force-displacement relationships given in Eqs. (20, 21, 23-26, 28-33) the element stiffness equations in incremental form:

$\left(\left[k_{\mathrm{e}}\right]+\left[k_{\mathrm{g}}\right]\right)\{u\}=\{f\}$

where the force increment vector $\{f\}$ has been moved to the right-hand side for convenience, and $\{u\}$ is the displacement increment vector of the element,

$\{f\}^{T}$

$$
\begin{array}{r}
=\left\langle F_{x A} F_{y A} F_{z A} M_{x A} M_{y A} M_{z A} F_{x B} F_{y B} F_{z B} M_{x B} M_{y B} M_{z B}\right\rangle \\
\{u\}^{T}=\left\langle u_{A} v_{A} w_{A} \theta_{x A} \theta_{y A} \theta_{z A} u_{B} v_{B} w_{B} \theta_{x B} \theta_{y B} \theta_{z B}\right\rangle
\end{array}
$$

The elastic stiffness matrix $\left[k_{\mathrm{e}}\right]$ as derived here is identical to the one commonly used in linear analysis [9], i.e.,

$\left[k_{\mathrm{e}}\right]=\left[\begin{array}{cccc}{\left[k_{1}\right]} & {\left[k_{2}\right]} & -\left[k_{1}\right] & {\left[k_{2}\right]} \\ & {\left[k_{3}\right]} & -\left[k_{2}\right]^{T} & {\left[k_{4}\right]} \\ & & {\left[k_{1}\right]} & -\left[k_{2}\right] \\ \text { Symm. } & & {\left[k_{3}\right]}\end{array}\right]$

where

$$
\begin{aligned}
& {\left[k_{1}\right]=\left[\begin{array}{lll}
E A / L & 0 & 0 \\
0 & 12 E I_{z} / L^{3} & 0 \\
0 & 0 & 12 E I_{y} / L^{3}
\end{array}\right]}
\end{aligned}
$$

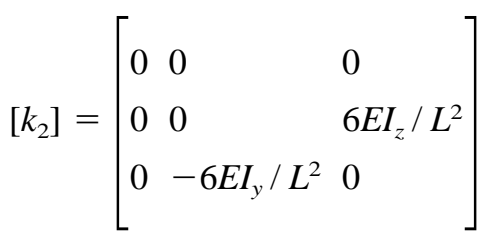

$$
\begin{aligned}
& {\left[k_{3}\right]=\left[\begin{array}{lll}
G J / L & 0 & 0 \\
0 & 4 E I_{y} / L & 0 \\
0 & 0 & 4 E I_{z} / L
\end{array}\right]} \\
& {\left[k_{4}\right]=\left[\begin{array}{lll}
-G J / L & 0 & 0 \\
0 & 2 E I_{y} / L & 0 \\
0 & 0 & 2 E I_{z} / L
\end{array}\right]}
\end{aligned}
$$


The geometric stiffness matrix $\left[k_{\mathrm{g}}\right]$ is more compact than those existing in the literature, which can be given as follows:

$$
\left[k_{\mathrm{g}}\right]=\left[\begin{array}{llll}
{[g]} & {\left[h_{A}\right]} & -[g] & {\left[h_{B}\right]} \\
{\left[h_{A}\right]^{T}} & {\left[i_{A}\right]} & -\left[h_{A}\right]^{T} & {[0]} \\
-[g] & -\left[h_{A}\right] & {[g]} & -\left[h_{B}\right] \\
{\left[h_{B}\right]^{T}} & {[0]} & -\left[h_{B}\right]^{T} & {\left[i_{B}\right]}
\end{array}\right]
$$

where use has been made of the relation ${ }^{1} M_{x A}=-$ ${ }^{1} M_{x B}$, and [0] is a $3 \times 3$ null matrix containing all zero entries,

$$
\begin{aligned}
{[g] } & =\left[\begin{array}{lll}
0 & -{ }^{1} F_{y B} / L & -{ }^{1} F_{z B} / L \\
-{ }^{1} F_{y B} / L & { }^{1} F_{x B} / L & 0 \\
-{ }^{1} F_{z B} / L & 0 & { }^{1} F_{x B} / L
\end{array}\right] \\
{\left[h_{B}\right] } & =\left[\begin{array}{lll}
0 & 0 & 0 \\
{ }^{1} M_{y B} / L & -{ }^{1} M_{x B} / 2 L & 0 \\
{ }^{1} M_{z B} / L & 0 & -{ }^{1} M_{x B} / 2 L
\end{array}\right] \\
{\left[i_{B}\right] } & =\left[\begin{array}{lll}
0 & 0 & 0 \\
-{ }^{1} M_{z B} & 0 & { }^{1} M_{x B} / 2 \\
{ }^{1} M_{y B} & -{ }^{1} M_{x B} / 2 & 0
\end{array}\right]
\end{aligned}
$$

and the submatrices $\left[h_{A}\right]$ and $\left[i_{A}\right]$ associated with end $A$ can be obtained by replacing the initial moments involved in the submatrices $\left[h_{B}\right]$ and $\left[i_{B}\right]$ of end $B$ by the corresponding ones associated with end $A$, namely, by replacing the terms $\left({ }^{1} M_{x B},{ }^{1} M_{y B},{ }^{1} M_{z B}\right)$ by $\left({ }^{1} M_{x A},{ }^{1} M_{y A}\right.$, $\left.{ }^{1} M_{z A}\right)$. For the special case of $2 \mathrm{D}$ beams, the elastic and geometric stiffness matrices are given in Appendix A.

Of interest is that the element geometric stiffness matrix $\left[k_{\mathrm{g}}\right]$ is asymmetric, as indicated by the induced moment matrix $\left[i_{B}\right]$, which relates to the nature of nodal moments undergoing 3D rotations [2]. The asymmetry in $\left[k_{\mathrm{g}}\right]$ can be attributed to the lack of conjugateness between the bending moments $M_{y}$ and $M_{z}$ and their corresponding displacement derivatives $-w^{\prime}\left(=\theta_{y}\right)$ and $v^{\prime}$ $\left(=\theta_{z}\right)$ that have been selected as the control DOFs of the element. For an in-depth discussion on selection of consistent rotational DOFs for large deformation analysis, the readers should be referred to the classic work of Reissner [10]. In this paper, however, we choose to use the displacement derivatives as the rotational DOFs, simply because they have been commonly adopted by structural analysts. The asymmetry of the $\left[k_{\mathrm{g}}\right]$ matrix presents no computation problem, since it is restricted to the element level, but not on the structure level, as will be demonstrated below.

\section{Equilibrium conditions for joints involving rotational DOFs}

It will be demonstrated that whenever the conditions of equilibrium are enforced for each node of the structure, the antisymmetric parts of the geometric stiffness matrices of all elements meeting at the same node cancel out. Consequently, the symmetry of the stiffness matrix is maintained for the structure $[1,2,9]$. For the present purpose, we shall concentrate on the submatrices $\left[i_{A}\right]$ and $\left[i_{B}\right]$ of the $\left[k_{\mathrm{g}}\right]$ matrix, as they are the source of asymmetry. Also, we shall restrict our discussion to node $B$, realizing that the conclusion applies to node $A$ of the element as well. For clarity, the subscripts ' $A$ ' and ' $B$ ' will just be dropped.

As shown in Fig. 6, consider an element with an initial moment vector ${ }^{1} \vec{M}$ acting at node $B$ in $C_{1}$, which can be expressed with reference to the local axes $x y z$ or global axes $X Y Z$ as

$$
\begin{aligned}
{ }^{1} \vec{M} & ={ }^{1} M_{x} \vec{e}_{x}+{ }^{1} M_{y} \vec{e}_{y}+{ }^{1} M_{z} \vec{e}_{z} \text { or }{ }^{1} \vec{M} \\
& ={ }^{1} M_{X} \vec{e}_{X}+{ }^{1} M_{Y} \vec{e}_{Y}+{ }^{1} M_{Z} \vec{e}_{Z}
\end{aligned}
$$

where $\vec{e}_{x}, \vec{e}_{y}, \vec{e}_{z}$ and $\vec{e}_{X}, \vec{e}_{Y}, \vec{e}_{Z}$ are the associated unit base vectors. The unit base vectors in the local axes can be related to those in the global axes as

$\vec{e}_{x}=l_{x} \vec{e}_{X}+m_{x} \vec{e}_{Y}+n_{x} \vec{e}_{Z}$

$\vec{e}_{y}=l_{y} \vec{e}_{X}+m_{y} \vec{e}_{Y}+n_{y} \vec{e}_{Z}$

$\vec{e}_{z}=l_{z} \vec{e}_{X}+m_{z} \vec{e}_{Y}+n_{z} \vec{e}_{Z}$

where $l_{x}, m_{x}, n_{x}, \ldots$, etc., are direction cosines. By the relations: $\vec{e}_{x}=\vec{e}_{y} \times \vec{e}_{z}, \quad \vec{e}_{y}=\vec{e}_{z} \times \vec{e}_{x}$ and $\vec{e}_{z}=$ $\vec{e}_{x} \times \vec{e}_{y}$, the following equalities can be derived:
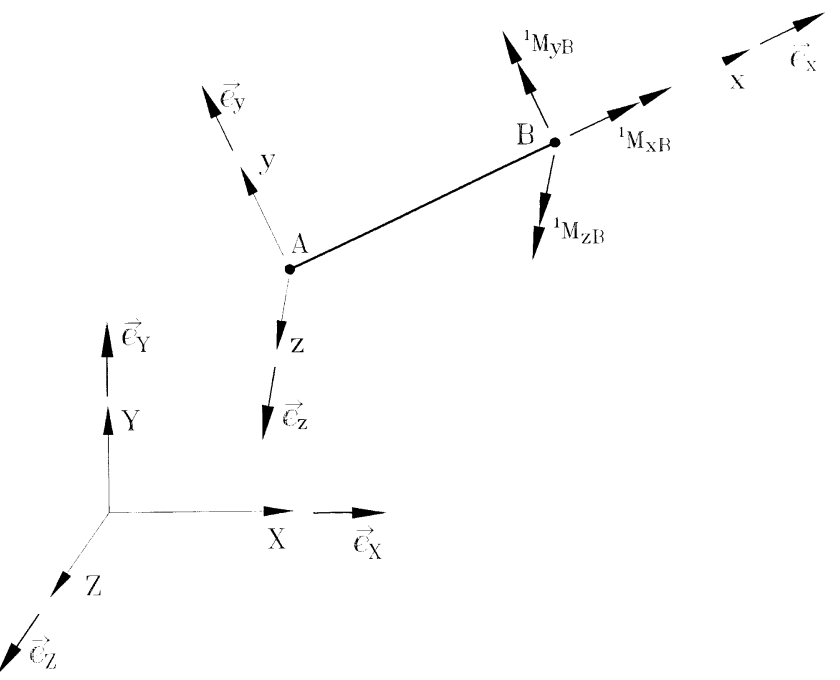

Fig. 6. Relation between the element axes and global axes. 
$l_{x}=m_{y} \cdot n_{z}-m_{z} \cdot n_{y} ; m_{x}=n_{y} \cdot l_{z}-n_{z} \cdot l_{y} ; n_{x}=l_{y} \cdot m_{z}-l_{z} \cdot m_{y}$

$l_{y}=m_{z} \cdot n_{x}-m_{x} \cdot n_{z} ; m_{y}=n_{z} \cdot l_{x}-n_{x} \cdot l_{z} ; n_{y}=l_{z} \cdot m_{x}-l_{x} \cdot m_{z}(45)$

$l_{z}=m_{x} \cdot n_{y}-m_{y} \cdot n_{x} ; m_{z}=n_{x} \cdot l_{y}$

$-n_{y} \cdot l_{x} ; n_{z}=l_{x} \cdot m_{y}-l_{y} \cdot m_{x}$

Let us decompose the induced moment matrix [i], which relates to the nature of moments undergoing rotations, into a symmetric part $[s]$ and an antisymmetric part $[a]$ :

$$
\begin{aligned}
& {[s]=\frac{1}{2}\left[\begin{array}{lll}
0 & -{ }^{1} M_{z} & { }^{1} M_{y} \\
-{ }^{1} M_{z} & 0 & 0 \\
{ }^{1} M_{y} & 0 & 0
\end{array}\right]} \\
& {[a]=\frac{1}{2}\left[\begin{array}{lll}
0 & { }^{1} M_{z} & -{ }^{1} M_{y} \\
-{ }^{1} M_{z} & 0 & { }^{1} M_{x} \\
{ }^{1} M_{y} & -{ }^{1} M_{x} & 0
\end{array}\right]}
\end{aligned}
$$

The key issue here is to show that when the antisymmetric component $[a]$ of the element is transformed from the local axes $x y z$ to the global axes $X Y Z$ and assembled with those of the other elements connected to the same node, they will just cancel each other. To this end, let us define the transformation matrix $[R]$ for relating the moment vector $\{m\}$ in the local axes to the one $\{M\}$ in the global axes:

$\{m\}=[R]\{M\} \quad$ or $\quad\{M\}=[R]^{T}\{m\}$

where

$$
\{m\}^{T}=\left\langle{ }^{1} M_{x}{ }^{1} M_{y}{ }^{1} M_{z}\right\rangle ; \quad\{M\}^{T}=\left\langle{ }^{1} M_{X}{ }^{1} M_{Y}{ }^{1} M_{z}\right\rangle
$$

and

$[R]$

$$
=\left[\begin{array}{llll}
\vec{e}_{x} \cdot \vec{e}_{X} & \vec{e}_{x} \cdot \vec{e}_{Y} & \vec{e}_{x} \cdot \vec{e}_{Z} \\
\vec{e}_{y} \cdot \vec{e}_{X} & \vec{e}_{y} \cdot \vec{e}_{Y} & \vec{e}_{y} \cdot \vec{e}_{Z} \\
\vec{e}_{z} \cdot \vec{e}_{X} & \vec{e}_{z} \cdot \vec{e}_{Y} & \vec{e}_{z} \cdot \vec{e}_{Z}
\end{array}\right] \equiv\left[\begin{array}{ccc}
l_{x} & m_{x} & n_{x} \\
l_{y} & m_{y} & n_{y} \\
l_{z} & m_{z} & n_{z}
\end{array}\right] \equiv\left[\begin{array}{c}
\vec{e}_{x} \\
\vec{e}_{y} \\
\vec{e}_{z}
\end{array}\right]
$$

Correspondingly, the antisymmetric component $[a]$ can be transformed to the global axes in the form of a triple product,

$$
[A]=[R]^{T}[a][R]
$$

With the use of Eq. (46b) for $[a]$, Eq. (49) for $[R]$, the equalities in Eq. (45), and the transformation relations in Eq. (47), we can now carry out the triple multiplication in Eq. (50) to obtain the antisymmetric submatrix $[A]$ in the global axes as
$[A]=\left[\begin{array}{lll}0 & { }^{1} M_{Z} & -{ }^{1} M_{Y} \\ -{ }^{1} M_{Z} & 0 & { }^{1} M_{X} \\ { }^{1} M_{Y} & -{ }^{1} M_{X} & 0\end{array}\right]$

Thus, when the antisymmetric components $[A]$ of all elements meeting at the same node are summed together, as part of the finite element assembly process, we have for this particular node (to be exact, the rotational DOFs) the following:

$$
\sum[A]=\left[\begin{array}{lll}
0 & \sum{ }^{1} M_{Z} & -\sum{ }^{1} M_{Y} \\
-\sum{ }^{1} M_{Z} & 0 & \sum{ }^{1} M_{X} \\
\sum{ }^{1} M_{Y} & -\sum{ }^{1} M_{X} & 0
\end{array}\right]
$$

Since each node of the structure is said to be in equilibrium at $C_{1}$, as is the case in an incremental analysis with iterations performed for removing the unbalanced forces, all the summations in Eq. (52) should vanish, i.e., $\Sigma^{1} M_{X}=\Sigma^{1} M_{Y}=\Sigma^{1} M_{Z}=0$. Thus, we have shown that the antisymmetric parts of all the elements meeting at the same node cancel each other, whenever the conditions of equilibrium are enforced for the $C_{2}$ configuration.

\section{Rigid body characteristics of the element derived}

Let $\left\{{ }_{1}^{1} f\right\}$ and $\left\{{ }_{1}^{2} f\right\}$ denote the nodal forces acting on the element at $C_{1}$ and $C_{2}$, both with reference to the $C_{1}$ axes, as were depicted in Figs. 3 and 5, respectively. Namely,

$\left\{{ }_{1}^{1} f\right\}^{T}$

$$
=\left\langle{ }^{1} F_{x A}{ }^{1} F_{y A}{ }^{1} F_{z A}{ }^{1} M_{x A}{ }^{1} M_{y A}{ }^{1} M_{z A}{ }^{1} F_{x B}{ }^{1} F_{y B}{ }^{1} F_{z B}{ }^{1} M_{x B}{ }^{1} M_{y B}{ }^{1} M_{z B}\right\rangle
$$

and the force vector $\left\{\begin{array}{l}2 \\ 1\end{array}\right\}$ can be defined simply by switching the superscripts in Eq. (53) from ' 1 ' to ' 2 '. With substitution of the force increments $\{f\}$ by $\left\{{ }_{1}^{2} f\right\}-$ $\left\{{ }_{1}^{1} f\right\}$, one may rewrite the incremental element Eq. (37) as

$\left\{\begin{array}{l}2 \\ 1\end{array}\right\}=\left\{1{ }_{1}^{1} f\right\}+\left[k_{\mathrm{g}}\right]\{u\}+\left[k_{\mathrm{e}}\right]\{u\}$

in which the configuration of reference is $C_{1}$. A physical interpretation for Eq. (54) can be given as follows: The total loads $\left\{\begin{array}{l}2 \\ 1\end{array}\right\}$ acting on the element at $C_{2}$ are balanced by the combined action of the initial forces $\left\{{ }_{1}^{1} f\right\}$, the elastic force increments $\left[k_{\mathrm{e}}\right]\{u\}$, and the forces induced by the $\left[k_{\mathrm{g}}\right]$ matrix upon nodal displacements. The equations as given in Eq. (54) are general by nature, which should be able to cope with all kinds of displacements complying with the assumption of small increments made in derivation, including, the rigid body displacements in particular. 
For a 3D beam element, there exist six modes of rigid displacements, i.e., three rigid translations and three rigid rotations. The elastic stiffness matrix $\left[k_{\mathrm{e}}\right]$ is known to be rigid-body qualified, which will not induce any forces upon rigid body displacements, i.e., $\left[k_{\mathrm{e}}\right]\{u\}_{\mathrm{r}}=$ $\{0\}$, where $\{u\}_{\mathrm{r}}$ denotes a rigid body mode and $\{0\}$ a zero vector. It follows that for the rigid body displacements, the element Eq. (54) reduce to

$\left\{\begin{array}{l}2 \\ 1\end{array}\right\}_{\mathrm{r}}=\left\{{ }_{1}^{1} f\right\}+\left[k_{\mathrm{g}}\right]\{u\}_{\mathrm{r}}$

where a subscript ' $r$ ' has been added to $\left\{{ }_{1}^{2} f\right\}$ to signify that it is associated with the rigid body displacement. As was stated in [9], the preceding equation serves as a criterion for checking the capability of the geometric stiffness matrix $\left[k_{\mathrm{g}}\right]$ to deal with rigid body displacements. According to the rigid body rule [9,11], for an initially stressed element subjected to a rigid body rotation, all the forces initially acting on the element must rotate following the rigid rotation, while their magnitudes remain unchanged. An overall result is the preservation of equilibrium of the element in the displaced position.

As was shown in Fig. 3, the initially stressed element is in equilibrium under the action of the nodal forces $\left\{{ }_{1}^{1} f\right\}$ at $C_{1}$. The configuration of the element after rigid body rotation can be conceived as the one in Fig. 7. To examine the capability of the derived matrix $\left[k_{\mathrm{g}}\right]$ in dealing with the rigid body displacements, one may start by assuming a rigid body mode $\{u\}_{\mathrm{r}}$ for the element, say, a rigid rotation $\theta_{y \mathrm{r}}$ about the $y$ axis,

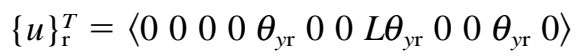

Then, one may substitute the rigid displacement $\{u\}_{\mathrm{r}}$ in Eq. (56), the $\left[k_{\mathrm{g}}\right]$ matrix in Eq. (41), and the initial element forces $\left\{\begin{array}{l}1 \\ 1\end{array}\right\}$ in Eq. (53) into the right-hand side of Eq. (55) to check if the resulting nodal forces $\left\{{ }_{1}^{2} f\right\}_{\mathrm{r}}$ really conform with the rigid body rule or the diagram given in Fig. 7. For the present case, it is easy to verify that the geometric stiffness $\left[k_{\mathrm{g}}\right]$ derived is qualified not

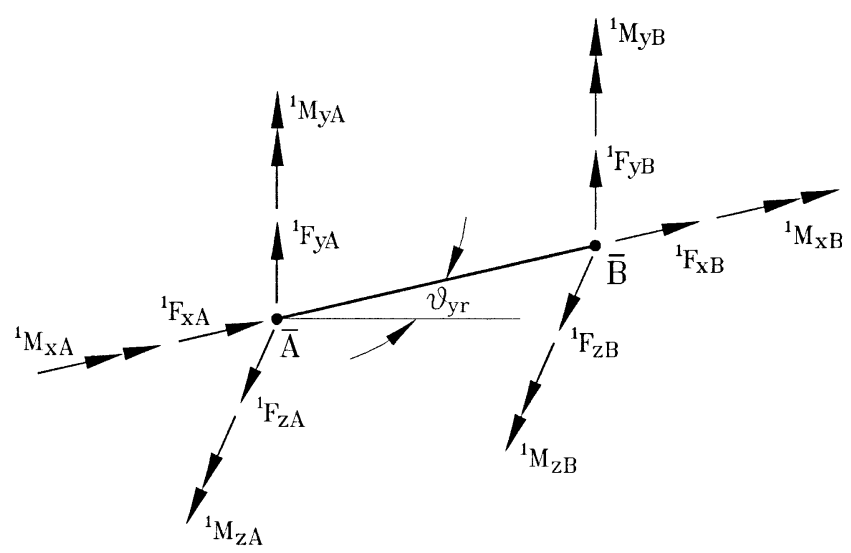

Fig. 7. Initially stressed beam after rigid rotation $\theta_{y r}$. only for the rigid rotation about the $y$ axis, but also for the other rigid body modes.

\section{Geometry updating and transformation matrices}

One essential step in the nonlinear analysis of 3D structural frames is the updating of orientation vectors for each element of the structure at the deformed configuration $C_{2}$, which serve as the basis for computing the transformation matrices. Consider the movement of an element from $C_{1}$ to $C_{2}$ in Fig. 8, where the orientation vectors $\left(\vec{e}_{x}, \vec{e}_{y}, \vec{e}_{z}\right)$ for the element at $C_{1}$ are assumed to be known. We shall focus on derivation of the orien-

tation vectors $\left(\vec{e}_{\bar{x}}, \vec{e}_{\bar{y}}, \vec{e}_{\bar{z}}\right)$ for the element at $C_{2}$ and the associated transformation matrix.

The transformation matrix $[\Delta R]$ for relating the $C_{1}$ to the $C_{2}$ axes is

$$
[\Delta R]=\left[\begin{array}{ccc}
\vec{e}_{\bar{x}} \cdot \vec{e}_{x} & \vec{e}_{\bar{x}} \cdot \vec{e}_{y} & \vec{e}_{\bar{x}} \cdot \vec{e}_{z} \\
\vec{e}_{\bar{y}} \cdot \vec{e}_{x} & \vec{e}_{\bar{y}} \cdot \vec{e}_{y} & \vec{e}_{\bar{y}} \cdot \vec{e}_{z} \\
\vec{e}_{\bar{z}} \cdot \vec{e}_{x} & \vec{e}_{z} \cdot \vec{e}_{y} & \vec{e}_{z} \cdot \vec{e}_{z}
\end{array}\right] \equiv\left[\begin{array}{c}
\Delta \vec{e}_{\bar{x}} \\
\Delta \vec{e}_{\bar{y}} \\
\Delta \vec{e}_{z}
\end{array}\right]
$$

Here, the incremental vectors $\left(\Delta \vec{e}_{x}, \Delta \vec{e}_{y}, \Delta \vec{e}_{z}\right)$ should be interpreted as the expressions of the $C_{2}$ vectors $\left(\vec{e}_{\bar{x}}\right.$, $\left.\vec{e}_{\bar{y}}, \vec{e}_{z}\right)$ with reference to the $C_{1}$ vectors $\left(\vec{e}_{x}, \vec{e}_{y}, \vec{e}_{z}\right)$. For the case of small displacements and small rotations within each incremental step, the preceding expression for $[\Delta R]$ reduces to the one given in Eq. (6), i.e.,

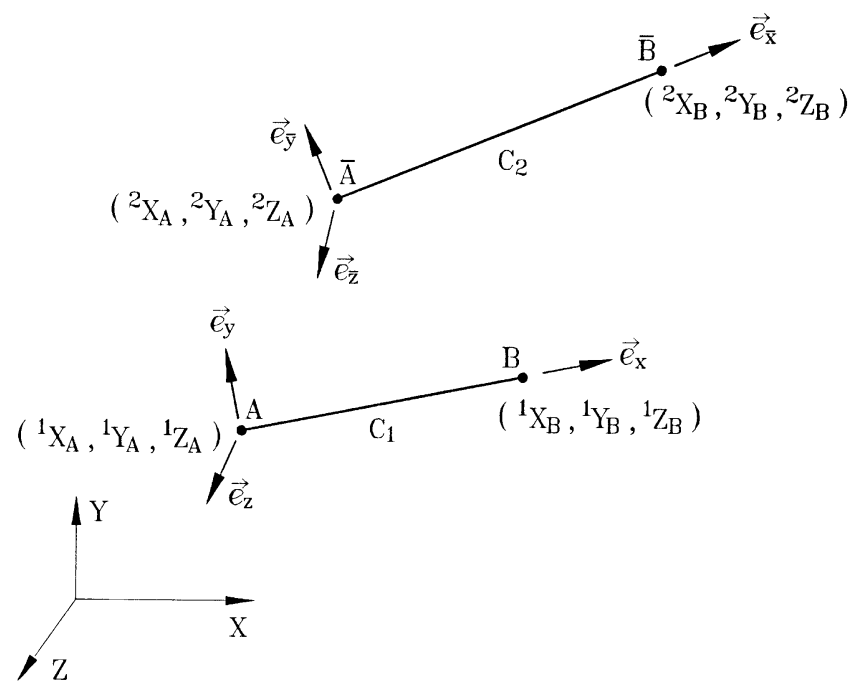

Fig. 8. Schematic of the movement of an element from $C_{1}$ to $C_{2}$. 
$[\Delta R] \cong\left[\begin{array}{lll}1 & \theta_{z \mathrm{r}} & -\theta_{y \mathrm{r}} \\ -\theta_{z \mathrm{r}} & 1 & \theta_{x \mathrm{r}} \\ \theta_{y \mathrm{r}} & -\theta_{x \mathrm{r}} & 1\end{array}\right] \equiv\left[\begin{array}{c}\Delta \vec{e}_{\alpha} \\ \Delta \vec{e}_{\beta} \\ \Delta \vec{e}_{\gamma}\end{array}\right]$

where $\theta_{x \mathrm{r}}, \theta_{y \mathrm{r}}, \theta_{\mathrm{zr}}$ denote the angles of rigid rotation of the element, as given in Eq. (4). The vectors $\left(\Delta \vec{e}_{\alpha}, \Delta \vec{e}_{\beta}\right.$, $\left.\Delta \vec{e}_{\gamma}\right)$ differ from the exact ones $\left(\Delta \vec{e}_{\bar{x}}, \Delta \vec{e}_{\bar{y}}, \Delta \vec{e}_{\bar{z}}\right)$ in that they are not unit vectors, and the conditions of orthogonality are violated. They are not qualified for use in updating the orientations of each element of the structure, simply because errors may be accumulated. In the following, we shall demonstrate how the vectors $\left(\Delta \vec{e}_{\alpha}\right.$, $\left.\Delta \vec{e}_{\beta}, \Delta \vec{e}_{\gamma}\right)$ can be corrected based on the conditions of orthogonality and normalization to yield the vectors $(\Delta$ $\left.\vec{e}_{\bar{x}}, \Delta \vec{e}_{\bar{y}}, \Delta \vec{e}_{\bar{z}}\right)$ desired.

Let $\left({ }^{2} X_{A},{ }^{2} Y_{A},{ }^{2} Z_{A}\right)$ and $\left({ }^{2} X_{B},{ }^{2} Y_{B},{ }^{2} Z_{B}\right)$ denote the coordinates of the two ends of the element at $C_{2}$. The vector directed along the axis of the element at $C_{2}$ is

$\vec{e}_{\bar{x}}=\frac{1}{L_{2}}\left\langle{ }^{2} X_{B}-{ }^{2} X_{A}{ }^{2} Y_{B}-{ }^{2} Y_{A}{ }^{2} Z_{B}-{ }^{2} Z_{A}\right\rangle$

where

$L_{2}=\sqrt{\left({ }^{2} X_{B}-{ }^{2} X_{A}\right)^{2}+\left({ }^{2} Y_{B}-{ }^{2} Y_{A}\right)^{2}+\left({ }^{2} Z_{B}-{ }^{2} Z_{A}\right)^{2}}$

Consequently, the vector $\Delta \vec{e}_{\bar{x}}$ can be computed from Eq. (57) as

$\Delta \vec{e}_{\bar{x}}=\left\langle c_{x} c_{y} c_{z}\right\rangle$

where the components $c_{x}, c_{y}, c_{z}$ are

$c_{x}=\vec{e}_{\bar{x}} \cdot \vec{e}_{x} ; c_{y}=\vec{e}_{\bar{x}} \cdot \vec{e}_{y} ; c_{z}=\vec{e}_{\bar{x}} \cdot \vec{e}_{z}$

given the unit base vectors $\left(\vec{e}_{x}, \vec{e}_{y}, \vec{e}_{z}\right)$ for the element at $C_{1}$. Next, let $\theta_{x A}$ and $\theta_{x B}$ denote the angles of twisting of the element at the two ends with respect to the $C_{1}$ axes. The angle of rigid twisting $\theta_{x \mathrm{r}}$ of the element can be computed from Eq. (4a). Consequently, the vectors $\Delta \vec{e}_{\beta}$ and $\Delta \vec{e}_{\gamma}$ can be obtained from Eq. (58) as

$\Delta \vec{e}_{\beta}=\left\langle-c_{y} 1 \theta_{x r}\right\rangle ; \Delta \vec{e}_{\gamma}=\left\langle-c_{z}-\theta_{x r} 1\right\rangle$

In the following, the two vectors $\Delta \vec{e}_{\beta}, \Delta \vec{e}_{\gamma}$ will be orthogonalized and normalized. First, they can be made orthogonal to the axial vector $\Delta \vec{e} \vec{x}_{\text {: }}$

$\vec{e}^{\prime}{ }_{b}=\frac{1}{\mu_{b}} \vec{e}_{b}{ }_{b} ; \vec{e}_{c}=\frac{1}{\mu_{c}} \vec{e}_{c}$

where
$\vec{e}_{b}^{\prime}=\Delta \vec{e}_{\beta}-\left(\Delta \vec{e}_{\beta} \cdot \Delta \vec{e}_{x}\right) \Delta \vec{e}_{x} ; \quad \vec{e}_{c}^{\prime}=\Delta \vec{e}_{\gamma}-\left(\Delta \vec{e}_{\gamma}{ }_{\gamma} \Delta \vec{e}_{\bar{e}}\right) \Delta \vec{e}_{\bar{x}}$

$\mu_{b}=\left|\vec{e}^{\prime}{ }_{b}\right| ; \quad \mu_{c}=\left|\vec{e}^{\prime}{ }_{c}\right|$

The two vectors are normal to the axial vector $\Delta \vec{e}_{\bar{x}}$, but are not orthogonal to each other. For this, further correction is required. Let

$\Delta \vec{e}_{\bar{y}}=\frac{1}{\mu_{1}}\left(\vec{e}_{b}+\mu_{2} \vec{e}_{c}\right) ; \quad \Delta \vec{e}_{\bar{z}}=\frac{1}{\mu_{1}}\left(\vec{e}_{c}+\mu_{2} \vec{e}_{b}\right)$

where the two coefficients $\mu_{1}, \mu_{2}$ are to be determined. Because the two vectors $\Delta \vec{e}_{\beta}, \Delta \vec{e}_{\gamma}$ are normal to the axial vector $\Delta \vec{e}_{\bar{x}}$, so are the two vectors $\Delta \vec{e}_{\bar{y}}, \Delta \vec{e}_{\bar{z}}$. By the orthogonality condition, $\Delta \vec{e}_{\bar{y}} \cdot \Delta \vec{e}_{\bar{z}}=0$, it can be solved

$\mu_{2}=\frac{1}{\vec{e}_{b} \cdot \vec{e}_{c}}\left(-1+\sqrt{1-\left(\vec{e}_{b} \cdot \vec{e}_{c}\right)^{2}}\right)$

Also, by the normalization condition, $\Delta \vec{e}_{\bar{y}} \cdot \Delta \vec{e}_{\bar{y}}=1$, the other coefficient can be solved,

$\mu_{1}=\sqrt{1+\mu_{2}^{2}+2 \mu_{2}\left(\vec{e}_{b} \cdot \vec{e}_{c}\right)}$

With the coefficients $\mu_{1}, \mu_{2}$ determined, the two vectors $\Delta \vec{e}_{\bar{y}}, \Delta \vec{e}_{\bar{z}}$, can be computed from Eq. (66).

As the three vectors $\left(\Delta \vec{e}_{\bar{x}}, \Delta \vec{e}_{\bar{y}}, \Delta \vec{e}_{\bar{z}}\right)$ have been made available, the transformation matrix $[\Delta R]$ for relating the $C_{1}$ to the $C_{2}$ axes can be computed from Eq. (57) as

$[\Delta R]=\left[\begin{array}{c}\Delta \vec{e}_{\bar{x}} \\ \Delta \vec{e}_{\bar{y}} \\ \Delta \vec{e}_{\bar{z}}\end{array}\right]$

Accordingly, the transformation matrix $[\bar{R}]$ for relating the $C_{2}$ axes of the element to the global axes $X Y Z$ of the structure is

$[\bar{R}]=[R][\Delta R]$

where the transformation matrix $[R]$ for relating the $C_{1}$ axes of the element to the global axes $X Y Z$ is known.

The orientation vectors $\left(\vec{e}_{\bar{x}}, \vec{e}_{\bar{y}}, \vec{e}_{\bar{z}}\right)$ of the element at $C_{2}$ can be obtained directly from the definition of the transformation matrix $[\bar{R}]$ as

$[\bar{R}]=\left[\begin{array}{lll}\vec{e}_{\bar{x}} \cdot \vec{e}_{X} & \vec{e}_{\bar{x}} \cdot \vec{e}_{Y} & \vec{e}_{\bar{x}} \cdot \vec{e}_{Z} \\ \vec{e}_{\bar{y}} \cdot \vec{e}_{X} & \vec{e}_{\bar{y}} \cdot \vec{e}_{Y} & \vec{e}_{\bar{y}} \cdot \vec{e}_{Z} \\ \vec{e}_{\bar{z}} \cdot \vec{e}_{X} & \vec{e}_{z} \cdot \vec{e}_{Y} & \vec{e}_{\bar{z}} \cdot \vec{e}_{Z}\end{array}\right] \equiv\left[\begin{array}{c}\vec{e}_{\bar{x}} \\ \vec{e}_{\bar{y}} \\ \vec{e}_{\bar{z}}\end{array}\right]$ 
As the orientation vectors $\left(\vec{e}_{\bar{x}}, \vec{e}_{\bar{y}}, \vec{e}_{\bar{z}}\right)$ and the transformation matrix $[\bar{R}]$ have been made available, one can proceed with the next iteration at each incremental step.

\section{Strategy for incremental-iterative analysis}

In performing the iteration during each incremental step, distinction is made between the predictor phase, concerning solution of the structural displacements $\{U\}$ from the structure equation $[K]\{U\}=\{P\}$, and the corrector phase for computing the element forces $\{f\}$ with reference to the $C_{2}$ axes, given the element displacements $\{u\}$. The former affects mostly the speed of convergence and the direction of iteration, while the latter the accuracy of solution. In this study, both the $\left[k_{\mathrm{e}}\right]$ and $\left[k_{\mathrm{g}}\right]$ matrices will be included in the predictor for constructing the structure matrix $[K]$, which is symmetric, as was explained in Section 3. However, only the $\left[k_{\mathrm{e}}\right]$ matrix will be considered in updating the element forces $\left\{{ }_{2}^{2} f\right\}$ at $C_{2}$ with reference to the same axes, i.e.,

$$
\left\{{ }_{2}^{2} f\right\}=\left\{{ }_{1}^{1} f\right\}+\left[k_{\mathrm{e}}\right]\{u\}
$$

where the first term on the right-hand side relates to the rigid body effect of the initial nodal forces according to the rigid body rule, and the second term the elastic deformation effect. Notice that exclusion of the $\left[k_{\mathrm{g}}\right]$ matrix from the deformation effect conforms to the assumption of small strains, small displacements, and small rotations within each incremental step.

\section{Numerical examples}

The element matrices derived in this paper, together with the strategy described in the preceding section and the generalized displacement control (GDC) method [12], will be adopted in the numerical studies. The tool of computation is Pentium 100 PC with 16 MB RAM. For comparison, three different expressions will be tried each for the predictor and corrector in the numerical study, that is,

- Predictor: The structural stiffness matrix $[K]$ is assembled using either of the following:

(P1) $\left[k_{\mathrm{e}}\right]+\left[k_{\mathrm{g}}\right]^{*}$, where $\left[k_{\mathrm{g}}\right] *$ is the 'conventional' one given on p. 360 of Ref. [9];

(P2) $\left[k_{\mathrm{e}}\right]+\left[k_{\mathrm{g}}\right]$, where $\left[k_{\mathrm{g}}\right]$ is the one derived in this paper, i.e., Eq. (41); and

(P3) $\left[k_{\mathrm{e}}\right]$ matrix only.

- Corrector: The term $\left[k_{\mathrm{e}}\right]\{u\}$ in Eq. (71) represents the elastic force increments, which will be replaced by either of the following:

(C1) $\{f\}=\left(\left[k_{\mathrm{e}}\right]+\left[k_{\mathrm{g}}\right]^{*}\right)\{u\}_{\mathrm{n}}$, where $\{u\}_{\mathrm{n}}$ is the natural deformation part of $\{u\}$;
(C2) $\{f\}=\left(\left[k_{\mathrm{e}}\right]+\left[k_{\mathrm{g}}\right]\right)\{u\}_{\mathrm{n}}$; and

(C3) $\{f\}=\left[k_{\mathrm{e}}\right]\{u\}$ as proposed in Eq. (71) of this paper.

As can be seen, there is a total of 9 combinations for the predictor and corrector.

Example 1. The angled frame under uniform bending (i.e., with $M_{Z A}=M_{Z C}$ ) in Fig. 9 was studied by Argyris et al. [4] as a test of the capability of a finite element to maintain equilibrium of structural joints connecting non-aligned members in the buckling position. The following data have been used: $A=18 \mathrm{~mm}^{2}, L=240$ $\mathrm{mm}, E=71,240 \mathrm{~N} / \mathrm{mm}^{2}, \quad G=21,790 \mathrm{~N} / \mathrm{mm}^{2}, \quad J=$ $2.16 \mathrm{~mm}^{4}, I_{y}=0.54 \mathrm{~mm}^{4}$, and $I_{z}=1350 \mathrm{~mm}^{4}$. Due to symmetry of the angled frame, only the left half is analysed, which is modeled by ten elements. The end $A$ of the member is restrained against rotations about the $X$ and $Y$ axes and against translations along the $Y$ and $Z$ axes. When the bending moments reach the critical value, the frame will buckle laterally out of the $X Y$ plane. To initialize the lateral deflection of the frame in the present analysis, a shear load $F_{Z B}(\mathrm{~N})$ of magnitude $5 \times 10^{-5} M_{Z A}$ serving as an imperfection is applied at node $B$. The deflection of the apex $B$ of the frame solved by the GDC method using the three combinations of predictor and corrector, $\mathrm{P} 1 \mathrm{C} 1, \mathrm{P} 2 \mathrm{C} 2$, and $\mathrm{P} 3 \mathrm{C} 3$, has been plotted against the load for both positive and negative bending in Figs. 10 and 11, respectively. As can be seen, basically no difference can be made among the results solved using either set of predictor and corrector, and the limit-point load corresponds very well to the theoretical loads $M_{\text {cr }}= \pm \pi \sqrt{ }\left(E I_{z} G J\right) / L= \pm 622.2 \mathrm{~N}-\mathrm{mm}$ for the two cases considered.

For this problem, the total running time consumed by each set of predictor and corrector has been compiled in Tables 1 and 2 respectively for the frame in positive and negative bending. Note that the use of the $\left[k_{\mathrm{e}}\right]$ matrix alone in the predictor may result in nonconvergent solutions, due to its lack of capability in simulating the rigid body effect in iterations. On the other hand, although all three correctors $\mathrm{C} 1, \mathrm{C} 2$, and $\mathrm{C} 3$ can be used to yield

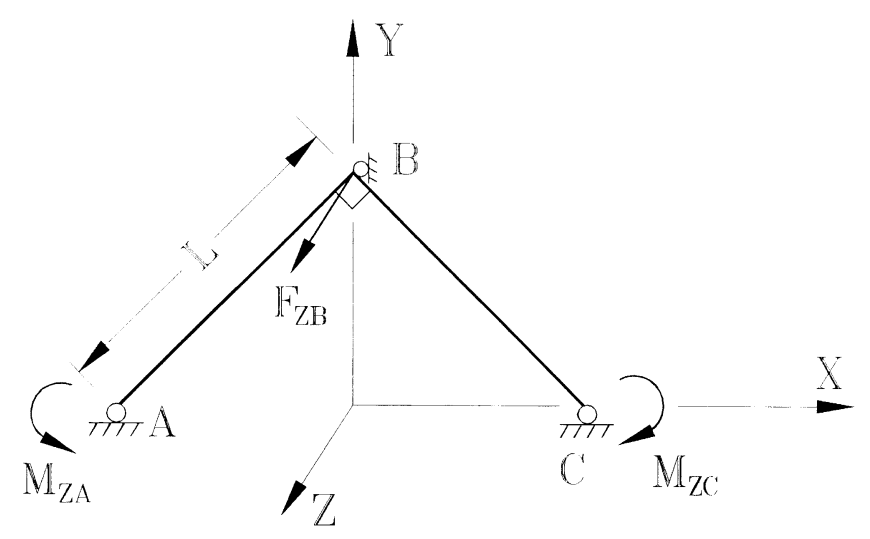

Fig. 9. Hinged angled frame in pure bending. 


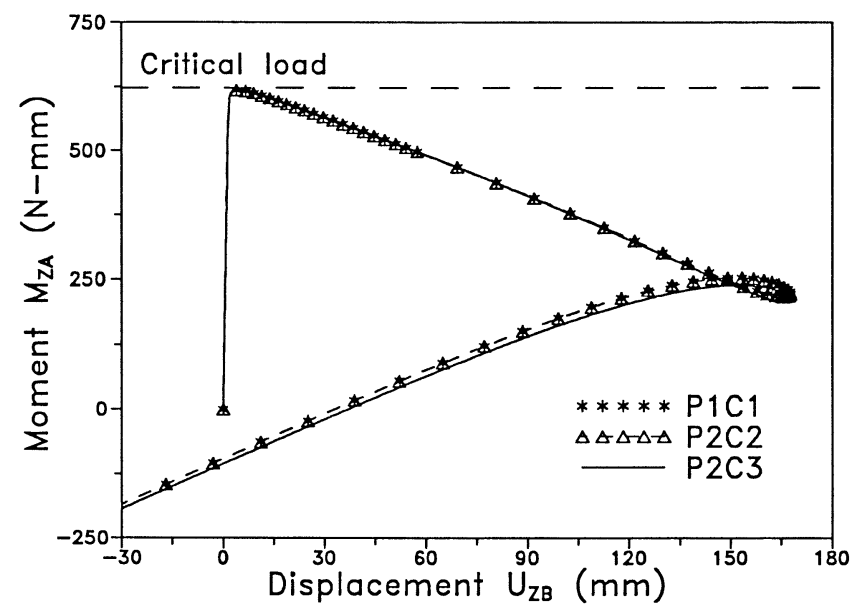

Fig. 10. Load-deflection curve for angled frame in positive bending.

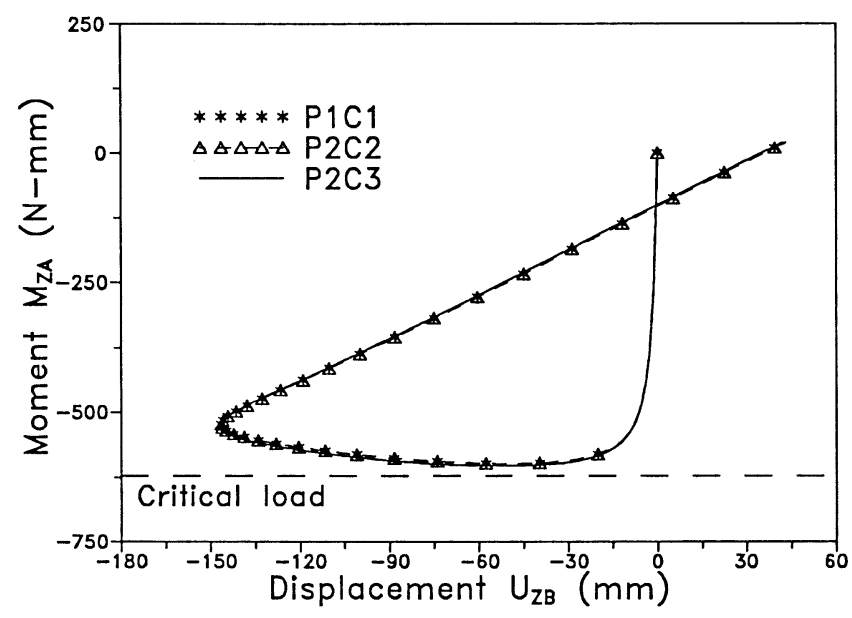

Fig. 11. Load-deflection curve for angled frame in negative bending.

Table 1

Running time for analysing angled frame in positive bending (s) ${ }^{\mathrm{a}}$

\begin{tabular}{llll}
\hline & Corrector & & \\
Predictor & $\mathrm{C} 1$ & $\mathrm{C} 2$ & $\mathrm{C} 3$ \\
\hline $\mathrm{P} 1$ & 102.93 & 102.76 & 73.71 \\
$\mathrm{P} 2$ & 102.75 & 101.95 & 73.46 \\
$\mathrm{P} 3$ & $*$ & $*$ & $*$ \\
\hline \multirow{2}{*}{ a *Nonconvergent. }
\end{tabular}

Table 2

Running time for analysing angled frame in negative bending (s) ${ }^{\mathrm{a}}$

\begin{tabular}{llll}
\hline & Corrector & & \\
Predictor & C1 & C2 & C3 \\
\hline P1 & 55.42 & 55.14 & 37.57 \\
P2 & 55.13 & 54.76 & 37.43 \\
P3 & $*$ & $*$ & $*$ \\
\hline
\end{tabular}

a *Nonconvergent. accurate solutions, the use of corrector C3 is most efficient.

Example 2. The right-angled frame subjected to inplane load $F_{Y B}$ in Fig. 12 was also devised by Argyris et al. [4] as a benchmark problem. The same material and geometry data as those used in Example 1 were adopted. Each member of the frame was modeled by ten beam elements. To initialize the out-of-plane buckling deflection, an imperfection load $F_{Z B}$ equal to one thousandth of the in-plane load $F_{Y B}$ was applied. The load deflection curves solved using the three sets $\mathrm{P} 1 \mathrm{C} 1$, $\mathrm{P} 2 \mathrm{C} 2$, and $\mathrm{P} 3 \mathrm{C} 3$ for the tensile and compressive load cases were plotted in Figs. 13 and 14, respectively, from which one observes that all the incremental solutions are close to each other, corresponding well to the linearized critical loads of $1.088 \mathrm{~N}$ and $-0.6804 \mathrm{~N}$ respectively for the tension and compression cases [4]. From the computer running time listed in Tables 3 and 4 for all sets of predictor and corrector for the two cases, the superiority of the proposed scheme $\mathrm{P} 2 \mathrm{C} 3$ for iterative analysis is clearly illustrated.

Example 3. The third example studied is the curved beam with a subtended angle $120^{\circ}$ under uniform bending (i.e., with $M_{Z A}=M_{Z C}$ ) in Fig. 15. The material and geometry data adopted for this example are identical to those used in Example 1. To trigger the lateral buckling deformation, an imperfection load $F_{Z B}=5 \times 10^{-5} M_{Z A}$
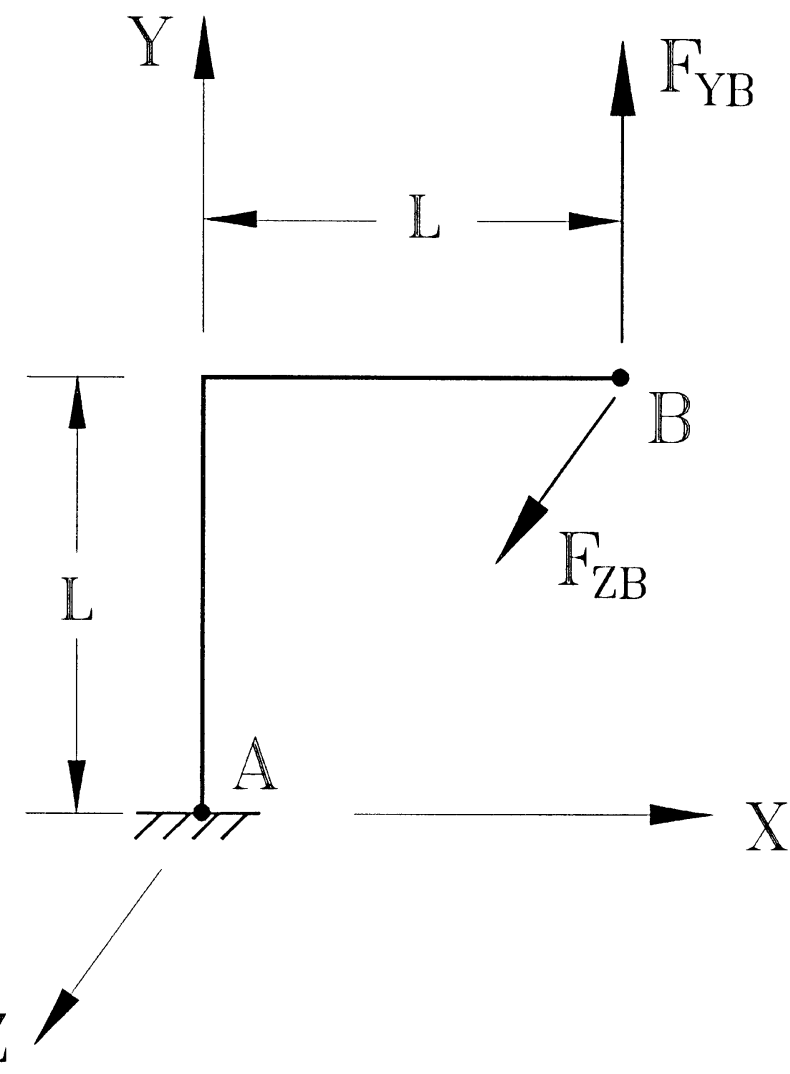

Fig. 12. Right angled frame with fixed support. 


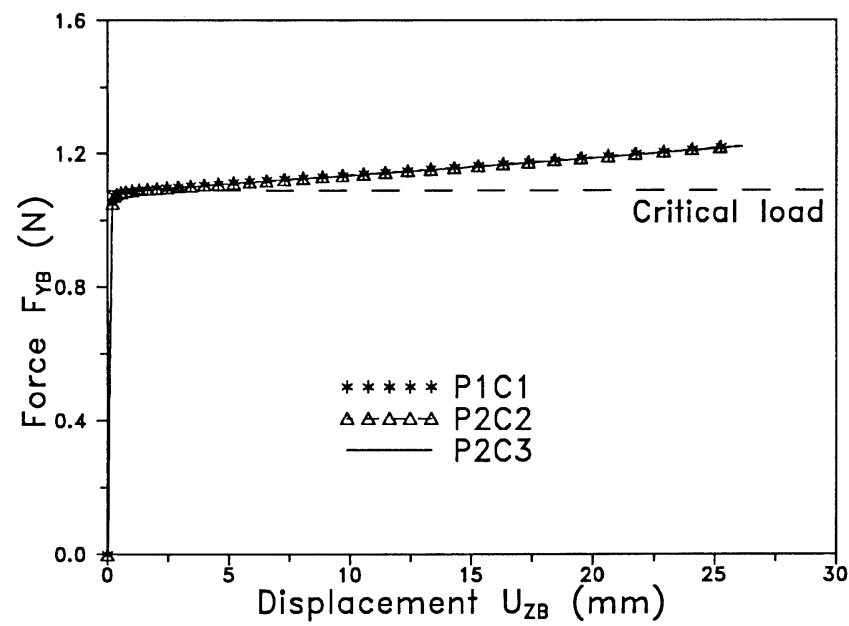

Fig. 13. Load-deflection curve for right angled frame in tension.

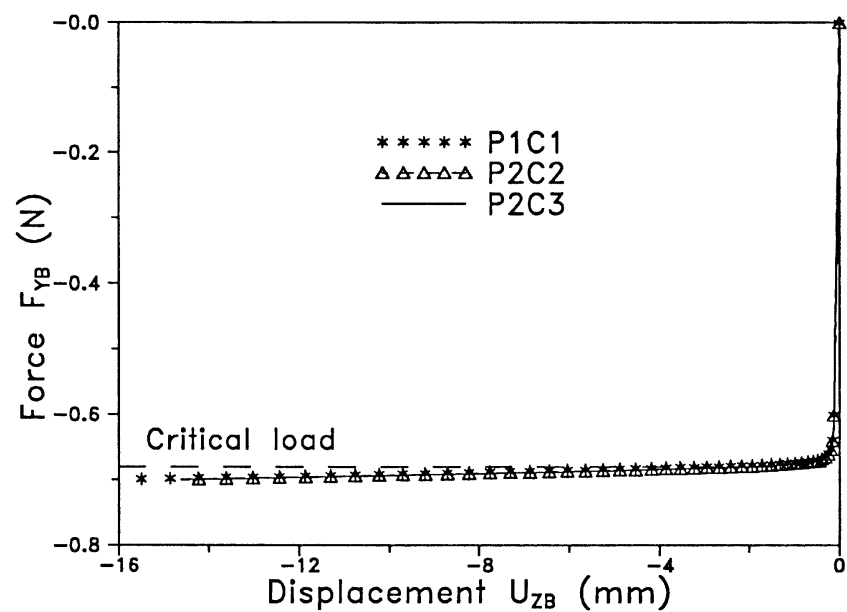

Fig. 14. Load-deflection curve for right angled frame in compression.

Table 3

Running time for analysing right angled frame in tension $(\mathrm{s})^{\mathrm{a}}$

\begin{tabular}{llll}
\hline & Corrector & & \\
Predictor & C1 & C2 & C3 \\
\hline P1 & 30.26 & 30.22 & 24.94 \\
P2 & 30.20 & 30.15 & 22.08 \\
P3 & $*$ & $*$ & $*$ \\
\hline
\end{tabular}

a *Nonconvergent.

Table 4

Running time for analysing right angled frame in compression (s)

\begin{tabular}{|c|c|c|c|}
\hline Predictor & $\begin{array}{l}\text { Corrector } \\
\mathrm{C} 1\end{array}$ & $\mathrm{C} 2$ & C3 \\
\hline P1 & 29.93 & 28.62 & 29.87 \\
\hline P2 & 35.05 & 28.46 & 27.14 \\
\hline P3 & 56.41 & 48.55 & 39.82 \\
\hline
\end{tabular}

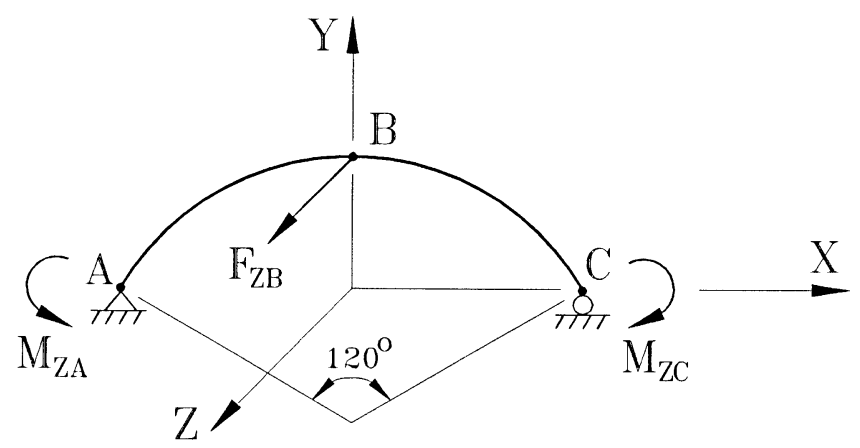

Fig. 15. Curved beam in uniform bending.

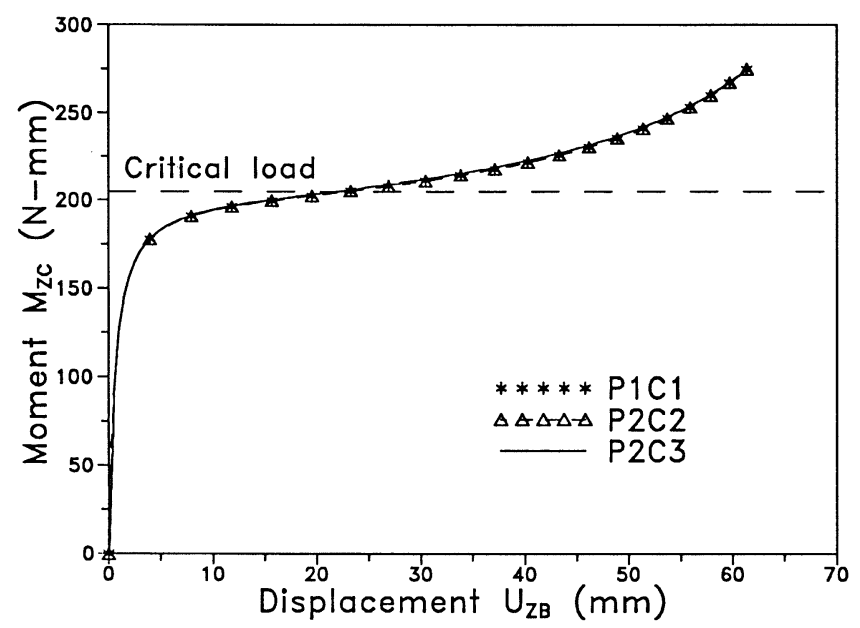

Fig. 16. Load-deflection curve for curved beam in positive bending.

was applied at point $B$. The circular length of the curved beam is assumed to be $L=240 \mathrm{~mm}$. The curved beam was approximated by ten chordwise beam elements. The load deflection curves solved using the three sets P1C1, P2C2, and P3C 3 have been plotted in Figs. 16 and 17 respectively for the beam in positive and negative bending, in which the critical loads, $M_{\mathrm{cr}}=204.8 \mathrm{~N}-\mathrm{mm}$,

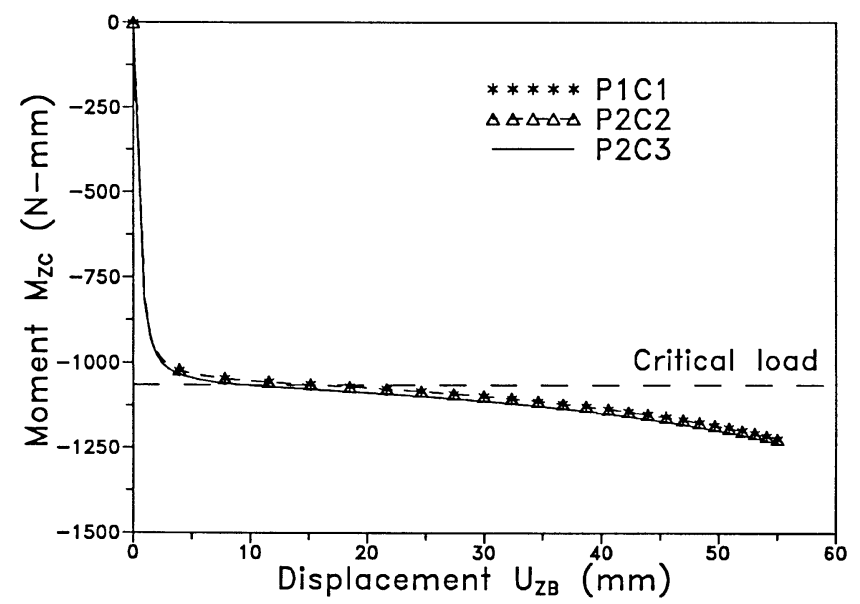

Fig. 17. Load-deflection curve for curved beam in negative bending. 
Table 5

Running time for analysing curved beam in positive bending (s)

\begin{tabular}{llll}
\hline & Corrector & & \\
Predictor & C1 & C2 & C3 \\
\hline P1 & 17.90 & 17.69 & 11.00 \\
P2 & 17.58 & 17.35 & 10.99 \\
P3 & 18.56 & 18.18 & 17.42 \\
\hline
\end{tabular}

$-1064.6 \mathrm{~N}-\mathrm{mm}$, obtained from the curved beam theory of Yang and Kuo (ch. 7 in ref. [9]) were also indicated. The computer running time for each analysis was listed in Tables 5 and 6 respectively for the positive and negative bending cases. From these tables, it is confirmed that the use of the proposed scheme $\mathrm{P} 2 \mathrm{C} 3$ can result in better efficiency for nonlinear analysis.

\section{Conclusions}

Strictly based on the assumption of small strains, small displacements and small rotations for each incremental step of the nonlinear analysis, the elastic and geometric stiffness matrices were derived for a 3D beam element. The geometric stiffness matrix is asymmetric on the element level, but is restored to be symmetric on the structure level, due to enforcement of joint equilibrium conditions at the deformed configuration. The present approach was referred to as a direct approach, since the element stiffness matrices were derived directly from the force-displacement relations of the incremental theory, which has the advantage of being physically interpretable compared with other established methods. The geometric stiffness matrix derived was demonstrated to be qualified for simulating the behaviors of initial nodal forces and moments undergoing 3D rotations. The element derived, along with the strategy of iteration, has been successfully employed to solve the nonlinear behavior of structural frames, with excursion into the postbuckling range. In terms of accuracy and efficiency, the present approach is just as competitive as any existing ones.

Table 6

Running time for analysing curved beam in negative bending (s) ${ }^{\mathrm{a}}$

\begin{tabular}{llll}
\hline & Corrector & & \\
Predictor & C1 & C2 & C3 \\
\hline P1 & 21.09 & 21.14 & 19.05 \\
P2 & 21.09 & 20.98 & 19.01 \\
P3 & $*$ & $*$ & $*$ \\
\hline
\end{tabular}

a *Nonconvergent.

\section{Acknowledgements}

The research reported herein is partly sponsored by the National Science Council of the Republic of China through Grant No. NSC87-2211-E-002-031.

\section{Appendix A. Reduction to 2D case}

For a 2D beam element with six DOFs at each node, the incremental stiffness equation is the same as the one given in Eq. (37). However, the force increment vector $\{f\}$ and displacement increment vector $\{u\}$ should be interpreted as

$$
\begin{aligned}
& \{f\}^{T}=\left\langle F_{x A} F_{y A} M_{z A} F_{x B} F_{y B} M_{z B}\right\rangle \\
& \{u\}^{T}=\left\langle u_{A} v_{A} \theta_{z A} u_{B} v_{B} \theta_{z B}\right\rangle
\end{aligned}
$$

The elastic stiffness matrix $\left[k_{\mathrm{e}}\right]$ is

$$
\left[k_{\mathrm{e}}\right]=\left[\begin{array}{ccccc}
\frac{E A}{L}{ }_{0} & 0 & -\frac{E A}{L} & 0 & 0 \\
\frac{12 E I_{z}}{L^{3}} & \frac{6 E I_{z}}{L^{2}} & 0 & -\frac{12 E I_{z}}{L^{3}} & \frac{6 E I_{z}}{L^{2}} \\
& \frac{4 E I_{z}}{L} & 0 & -\frac{6 E I_{z}}{L^{2}} & \frac{2 E I_{z}}{L} \\
& & \frac{E A}{L} & 0 & 0 \\
& & & \frac{12 E I_{z}}{L^{3}} & -\frac{6 E I_{z}}{L^{2}} \\
& & & & \frac{4 E I_{z}}{L}
\end{array}\right]
$$

which is the same as the one commonly known [9]. The geometric stiffness matrix $\left[k_{\mathrm{g}}\right]$ as shown below contains a number of zero terms,

$$
\left[k_{\mathrm{g}}\right]=\left[\begin{array}{ccccc}
\frac{{ }^{1} M_{z A}+{ }^{1} M_{z B}}{L^{2}} & 0 & 0 & -\frac{{ }^{1} M_{z A}+{ }^{1} M_{z B}}{L^{2}} & 0 \\
\frac{{ }^{1} F_{x B}}{L} & 0 & -\frac{{ }^{1} M_{z A}+{ }^{1} M_{z B}}{L^{2}} & -\frac{{ }^{1} F_{x B}}{L} & 0 \\
& 0 & 0 & 0 & 0 \\
& 0 & \frac{{ }^{1} M_{z A}+{ }^{1} M_{z B}}{L^{2}} & 0 \\
& & \frac{{ }^{1} F_{x B}}{L} & 0 \\
\text { Symm. } & & & 0
\end{array}\right]
$$

The fact that all entries in the third row are null implies that no bending moments will be induced by any rigid displacements. The $\left[k_{\mathrm{g}}\right]$ matrix is more compact than most existing ones. However, it has the desired property of being rigid body qualified. 


\section{References}

[1] Yang YB, Kuo SR. Consistent frame buckling analysis by finite element method. Journal of Structural Engineering, ASCE 1991;117(4):1053-69.

[2] Yang YB, Kuo SR. Frame buckling analysis with full consideration of joint compatibili ties. Journal of Engineering Mechanics, ASCE 1992;118(5):871-89.

[3] Argyris JH, Dunne PC, Malejannakis GA, Scharph DW. On large displacement small strain analysis of structures with rotational degrees of freedom. Computer Methods in Applied Mechanics and Engineering 1978;14:401-51, 15:99-135.

[4] Argyris JH, Hilbert O, Malejannakis GA, Scharph DW. On the geometrical stiffness of a beam in space a consistent v.w. approach. Computer Methods in Applied Mechanics and Engineering 1979;20:105-31.

[5] Elias ZM. Theory and methods of structural analysis. New York: John Wiley, 1986.
[6] Yang YB, McGuire W. Stiffness matrix for geometric nonlinear analysis. Journal of Structural Engineering, ASCE 1986;112(4):853-77.

[7] Yang YB, McGuire W. Joint rotation and geometric nonlinear analysis. Journal of Structural Engineering, ASCE 1986;112(4):879-905.

[8] Ziegler H. Principle of structural stability, 2nd ed. Stuttgart: Birkhauser Verlag, 1977.

[9] Yang YB, Kuo SR. Theory and analysis of nonlinear framed structures. Englewood Cliffs, NJ: Prentice-Hall, 1994.

[10] Reissner E. One one-dimensional large-displacement finite-strain beam theory. In: Studies in applied mathematics 1973; LII(2): The Massachusetts Institute of Technology.

[11] Yang YB, Chiou HT. Rigid body motion test for nonlinear analysis with beam elements. Journal of Engineering Mechanics, ASCE 1996;113(9):1404-19.

[12] Yang YB, Shieh MS. Solution method for nonlinear problems with multiple critical points. AIAA Journal 1990;28:2110-6. 